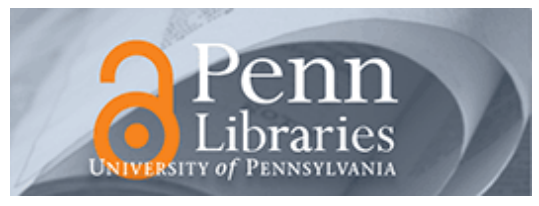

University of Pennsylvania

ScholarlyCommons

4-1998

\title{
Diffusing-Light Spectroscopies Beyond the Diffusion Limit: The Role of Ballistic Transport and Anisotropic Scattering
}

\author{
P. A. Lemieux \\ University of California, Los Angeles \\ M. U. Vera \\ University of California, Los Angeles \\ Douglas J. Durian \\ University of Pennsylvania, djdurian@physics.upenn.edu
}

Follow this and additional works at: https://repository.upenn.edu/physics_papers

Part of the Physics Commons

\section{Recommended Citation}

Lemieux, P. A., Vera, M. U., \& Durian, D. J. (1998). Diffusing-Light Spectroscopies Beyond the Diffusion Limit: The Role of Ballistic Transport and Anisotropic Scattering. Physical Review E, 57 (4), 4498-4515.

http://dx.doi.org/10.1103/PhysRevE.57.4498

At the time of publication, author Douglas J. Durian was affiliated with University of California, Los Angeles.

Currently, he is a faculty member at the Physics Department at the University of Pennsylvania.

This paper is posted at ScholarlyCommons. https://repository.upenn.edu/physics_papers/624

For more information, please contact repository@pobox.upenn.edu. 


\title{
Diffusing-Light Spectroscopies Beyond the Diffusion Limit: The Role of Ballistic Transport and Anisotropic Scattering
}

\begin{abstract}
Diffuse transmission and diffusing-wave spectroscopy (DWS) can be used to probe the structure and dynamics of opaque materials such as colloids, foams, and sand. A crucial step is to model photon transport as a diffusion process. This approach is acceptable for optically thick samples, far into the limit of strong multiple scattering; however, it becomes increasingly inaccurate for thinner samples for several reasons. Here, we correct for two of these defects. By modeling photon propagation by a telegrapher equation with suitable boundary conditions, we can account for the ballistic transport of photons at finite speed between successive scattering events. By introducing a discontinuity in the photon concentration at the source point, and then averaging over a range of penetration depths, we can account for the fact that photons usually scatter anisotropically into the forward direction, rather than being completely randomized at each event. The accuracy of our approach is tested by comparison both with random walk computer simulations and with experiments on specially designed suspensions of polystyrene spheres. We find that our predictions extend the utility of diffuse transmission to slabs of all thicknesses and of DWS to slabs down to about two transport mean free paths.

\section{Disciplines}

Physical Sciences and Mathematics | Physics

\section{Comments}

At the time of publication, author Douglas J. Durian was affiliated with University of California, Los Angeles. Currently, he is a faculty member at the Physics Department at the University of Pennsylvania.
\end{abstract}




\title{
Diffusing-light spectroscopies beyond the diffusion limit: The role of ballistic transport and anisotropic scattering
}

\author{
P.-A. Lemieux, M. U. Vera, and D. J. Durian \\ Department of Physics and Astronomy, University of California, Los Angeles, California 90095-1547
}

(Received 14 October 1997)

\begin{abstract}
Diffuse transmission and diffusing-wave spectroscopy (DWS) can be used to probe the structure and dynamics of opaque materials such as colloids, foams, and sand. A crucial step is to model photon transport as a diffusion process. This approach is acceptable for optically thick samples, far into the limit of strong multiple scattering; however, it becomes increasingly inaccurate for thinner samples for several reasons. Here, we correct for two of these defects. By modeling photon propagation by a telegrapher equation with suitable boundary conditions, we can account for the ballistic transport of photons at finite speed between successive scattering events. By introducing a discontinuity in the photon concentration at the source point, and then averaging over a range of penetration depths, we can account for the fact that photons usually scatter anisotropically into the forward direction, rather than being completely randomized at each event. The accuracy of our approach is tested by comparison both with random walk computer simulations and with experiments on specially designed suspensions of polystyrene spheres. We find that our predictions extend the utility of diffuse transmission to slabs of all thicknesses and of DWS to slabs down to about two transport mean free paths. [S1063-651X(98)11604-5]
\end{abstract}

PACS number(s): 82.70.- y, 05.40.+j, 42.62.Fi

\section{INTRODUCTION}

One of the hallmark features of soft-condensed matter is the presence of structure at length scales that are intermediate between the molecular and the macroscopic. Diverse examples include the arrangement of colloidal particles into liquid, glassy, or crystalline structures in paint, ink, and dairy products; the packing of gas or liquid bubbles inside foams and emulsions; the arrangement of grains in a pile of sand; or the configuration of cells in a tissue [1]. These mesoscopic structures are interesting both in terms of the microscopic physics underlying their formation, and in terms of the macroscopic properties they impart to the material. Since the individual components typically have different refractive indices, they can strongly scatter visible light and hence cause bulk samples to have an opaque, white appearance. Physically, photons travel ballistically between successive scattering events whose cumulative effect is to produce a random walk. This can preclude characterization by traditional means such as video microscopy or angle-resolved static and quasielastic light scattering. Fortunately, experimental techniques that exploit multiple light scattering have been developed for probing the structure and dynamics of such materials [2]. In diffuse-transmission spectroscopy (DTS), the transmission probability $T$ for incident light to be transmitted through an opaque slab is measured as a function of wavelength and analyzed in terms of the transport mean free path, $l^{*}$, or step size in the random walk, of the photons [3]. Structural details are then deduced from the value and wavelength dependence of $l^{*}$. In diffusing-wave spectroscopy (DWS), fluctuations in the intensity of a portion of the multiply scattered light are measured and expressed in terms of a normalized electric field autocorrelation function, $g_{1}(\tau)$ $=\left\langle E(0) E^{*}(\tau)\right\rangle /\left\langle|E|^{2}\right\rangle$, as a function of the delay time $\tau$ [4-6]. Results are then analyzed in terms of $\left\langle\Delta r^{2}(\tau)\right\rangle$, the mean-squared change in position of the scattering sites due to thermal motion, flow, or time evolution.

For analysis of both DTS and DWS data, most widely used theories approximate the propagation of multiply scattered photons as a diffusion process. This yields convenient analytic expressions that are reasonably accurate if the sample thickness is much greater than $l^{*}$, where the number of scattering events is large. Unfortunately, however, experiments cannot be performed on arbitrarily thick samples due to finite absorption and coherence lengths, and also due to considerations of the signal-to-noise ratio. Consequently, sample thickness is most often chosen in the range 5 $<L / l^{*}<20$, where the diffusion approximations are on the verge of being unacceptably inaccurate. For smaller thicknesses, the failure of diffusion theory predictions has been observed experimentally $[7,8]$. Furthermore, the effects of scattering anisotropy, where photons are not completely randomized at each scattering event (see Fig. 1), and ballistic propagation, where photons travel at a finite speed between events, can be important for thin samples. Because of the difficulty in accounting for such phenomena in theories of

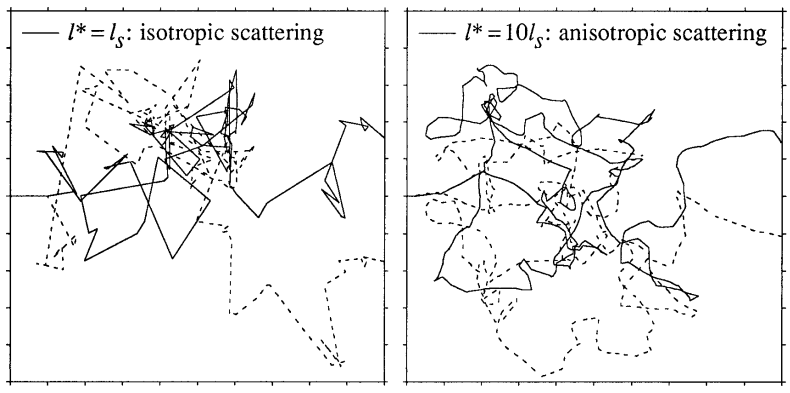

FIG. 1. Transverse coordinates of random walks across a threedimensional slab of thickness $101^{*}$, for isotropic and anisotropic scattering. In both cases, the walks start inward at the left and exit at the right. 
photon transport, multiple light scattering techniques have not yet achieved the same degree of quantitative accuracy as the traditional, single-scattering, techniques $[9,10]$.

Several methods have been proposed to improve upon the diffusion approximations for DWS. MacKintosh and John [11] described an integral formalism for dealing with correlations between scattering sites located closer than one transport mean free path, where the propagation of light is not diffusive and where wave effects are important. Their approach is also capable of dealing with the polarization effects observed in backscattering experiments [12]. In analogy with radiative transfer, a correlation transfer formalism has been proposed by Ackerson, Dougherty, and co-workers $[13,14]$ for extending the theory of DWS to thinner slabs. Both of these approaches, however, require either numerical solution or systematic expansion with the assumption that the diffusion approximation is nearly correct. An alternative approach by Middleton and Fisher [15] employs random-walk simulations, and hence avoids transport approximations altogether. Simulation results that demonstrate the limitations of diffusion approximations have been reported for both backscattering [15] and transmission [10] geometries. This approach is computationally intensive and does not yield closed-form predictions that can readily be used for data analysis.

Recently we have developed an exactly soluble theory of DTS and DWS that does not rely on diffusion approximations but instead models photon transport in terms of two counter-propagating streams [16]. This approach follows established procedures from the astrophysics literature for approximating transport in three dimensions from exact results for transport in a truly one-dimensional space. It provides a straightforward means of incorporating boundary reflectivity, scattering anisotropy, and ballistic propagation. The resulting predictions are more accurate, and have a greater range of validity, than those from diffusion theory. Unfortunately, however, the two-stream theory applies only to sample and illumination geometries that have a one-dimensional symmetry, has the wrong photon diffusion coefficient, and cannot account for the effects of angle-dependent wall reflectivity [16]. To address these problems, we have proposed an alternative means of generalizing upon the exact one-dimensional theory [17]. This yields a telegrapher equation for the total photon concentration, complete with specifications for the boundary conditions and the emerging flux. This theory reduces to standard diffusion theory for long times and distances, and in cases of weak absorption, where ballistic propagation is unimportant, and significantly improves upon it otherwise. Since the fundamental quantity is the total number of photons per volume, rather than per unit direction or per stream, it cannot account explicitly for scattering anisotropy.

In this paper, we report on how to incorporate scattering anisotropy into telegrapher and diffusion theories of photon transport. Physical arguments, and exact results from onedimensional transport, are used to demonstrate that a discontinuity in the total photon concentration at the source point is opened up in proportion to the degree of scattering anisotropy. Averaging such results over a continuous range of source points, to mimic an incident laser beam, leads to improved predictions for DTS and DWS. We begin in Secs. II and III, respectively, by reviewing the diffusion and telegra-

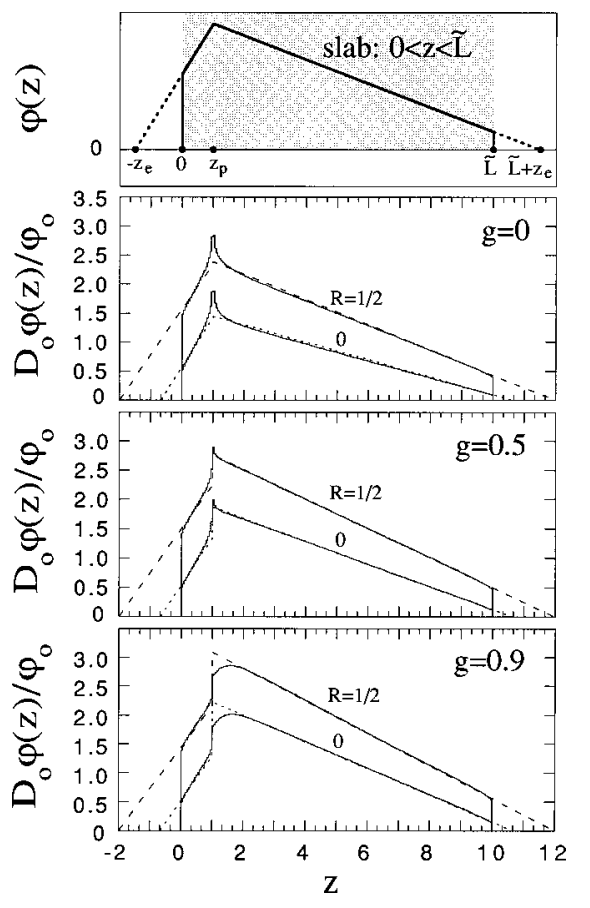

FIG. 2. Photon concentration vs distance into the sample for slabs of thickness $\widetilde{L}=10$ for photons that begin their random walks at $z_{p}=1$, where all lengths are measured in units of $l^{*}$, the photon transport mean free path. The top plot shows graphically the definitions of the penetration depth and extrapolation length ratios. In the bottom three plots, solid curves represent histogram data from random-walk simulations for different values of the average cosine of the scattering angle $g$, and boundary reflectivity $R$, as labeled. The dashed curves represent the predictions of Eq. (4.1), which has a discontinuity at $z_{p}$ and vanishes at $z=-z_{e}$ and $z=L+z_{e}$. The size of the discontinuity is fixed by the value of the transmission probability, and seen here to be given by $D_{0} \Delta \varphi \cong g$.

pher theories of photon transport. In Sec. IV, where new material starts, we argue that anisotropy is accounted for by a discontinuity at the source, and we develop diffusing-light spectroscopy predictions for slablike geometries. In Secs. V and VI, respectively, we test these predictions by randomwalk computer simulations and by experiment on dilute suspensions of colloidal particles.

\section{DIFFUSION APPROACH TO TRANSPORT}

The diffusion theory predictions for photon transport through a slab of thickness $L$ are summarized here, both for later comparison and to introduce notation. Throughout, the illumination and detection optics are taken such that there is no discrimination on the basis of the net lateral motion of photons within the sample, thus ensuring an effectively onedimensional symmetry. Ignoring absorption, three transport parameters enter into the theory: the photon transport mean free path $l^{*}$, defined such that the photon diffusion constant is $c l^{*} / 3$, the penetration depth $z_{p} l^{*}$, defined as the distance into the slab at which diffusing photons are assumed to be created, and the extrapolation length $z_{e} l^{*}$, defined as the distance outside the slab at which the diffuse photon concentration is assumed to extrapolate to zero. These definitions for the penetration depth and extrapolation length ratios are depicted graphically in the top plot of Fig. 2. The value of 
the transport mean free path is given by

$$
l^{*}=\frac{l_{s}}{\langle 1-\cos \theta\rangle}=\frac{l_{s}}{1-g},
$$

where $l_{s}$ is the scattering length, equal to the average distance between scattering events and also by which the intensity in a ballistic beam is exponentially attenuated, and $g$ $=\langle\cos \theta\rangle$ is the average cosine of the scattering angle $[18,19]$. The values of the penetration depth and extrapolation length ratios, $z_{p}$ and $z_{e}$, respectively specify the treatment of the source and boundary conditions, and are both of order one. In reality, diffusing photons are introduced into the sample over a continuous range of depths as photons scatter out of an incident beam; however, the penetration depth is usually set to its average value assuming the scattering to be isotropic: $z_{p}=1$. The extrapolation length ratio, on the other hand, is much better specified. It is set by the angle-dependent reflectivity $R_{w}(\mu)$ of the sample wall according to

$$
z_{e}=\frac{2}{3}\left(\frac{1+R_{2}}{1-R_{1}}\right) \quad \text { with } \quad R_{n}=\int_{0}^{1}(n+1) \mu^{n} R_{w}(\mu) d \mu,
$$

where the angle $\cos ^{-1} \mu$ is measured with respect to the interior normal $[20,21]$. Numerical results for many cases of experimental interest are given in Ref. [21] and confirmed by their connection to the angular dependence with which diffusely transmitted photons emerge from a slab.

For diffuse transmission spectroscopy, the average transmitted intensity is measured and analyzed in terms of the photon transport mean free path. Since the value of $l^{*}$ is determined by the nature and spatial arrangement of scattering sites, this gives a measure of the structure of the material. Given the above ingredients, the transmission probability for diffuse photons created at $z_{p} l^{*}$ is predicted by diffusion theory to be

$$
T_{z_{p}}=\frac{z_{p}+z_{e}}{\widetilde{L}+2 z_{e}}
$$

where $\widetilde{L}=L / l^{*}$ is the dimensionless optical thickness of the slab. This can be shown either by solution of the diffusion equation for a steady, time-independent source or by use of Green's function techniques. This result with $z_{p}=1$ is widely used to analyze experimental data. Random-walk simulations show that it describes the total diffuse transmission probability to within a few percent, for arbitrary scattering anisotropy and boundary reflectivity, as long as the sample thickness exceeds about $5 l^{*}$ and $z_{e}$ is taken according to Eq. (2.2) [9].

In diffusing-wave spectroscopy, fluctuations in the detected intensity are measured and then analyzed in terms of the time dependence of the dimensionless mean-squared displacement of the scattering sites, $x \equiv k^{2}\left\langle\Delta r^{2}(\tau)\right\rangle$ where $k$ is the wave vector of light inside the material. The normalized electric field autocorrelation function that describes these fluctuations is given by a weighted average of the single-path correlation function, $\exp (-x Y / 3)$, according to the probability density $P(Y)$ for the total dimensionless square wave vector transfer of the photon path to be

$$
Y \equiv \sum_{i=1}^{n} q_{i}^{2} / 2 k^{2}=\sum_{i=1}^{n}\left(1-\cos \theta_{i}\right)
$$

where $\mathbf{q}_{i}$ is the change in wave vector for scattering at site $i$ by angle $\theta_{i}$, for any total number $n$ of scattering events in the path $[4,15]$. This assumes that scattering events and particle motions are uncorrelated, and that $l^{*}$ is larger than the wavelength of light. The benchmark for predicting the detected correlation function is thus given by

$$
g_{1 Y}(x)=\int_{0}^{\infty} P(Y) e^{-x Y / 3} d Y
$$

Unfortunately, since the number of scattering events in a given path is discrete, this expression can only be evaluated by computer via random-walk simulations $[15,10]$. To make analytic progress, the so-called continuum approximation must be invoked to relate total square wave vector transfer to path length. If the number $n$ of scattering events in a path is large, then $Y$ may be approximated accurately by averaging over the scattering form factor:

$$
Y \cong n\langle 1-\cos \theta\rangle=\frac{n l_{s}}{l^{*}}=\frac{s}{l^{*}} \equiv S,
$$

where $s=S l^{*}$ is the total length of the light path. The approximate expression used to compute the normalized electric field autocorrelation function is thus

$$
g_{1 S}(x)=\int_{0}^{\infty} P(S) e^{-x S / 3} d S
$$

where $P(S)$ is the probability density for a detected photon to have a total path length of $S l^{*}$ inside the sample. Since the speed of light is constant, this expression is a Laplace transform with respect to time of the detected pulse resulting from an instantaneous source, and can thus be evaluated using Green's function techniques [5,2]. For the slab geometry with one-dimensional incident-collection optics, the correlation function in transmission is found to be

$$
g_{1 T, x_{p}}(x)=\frac{\sinh \left[z_{p} \sqrt{x}\right]+z_{e} \sqrt{x} \cosh \left[z_{p} \sqrt{x}\right]}{T_{z_{p}}\left\{\left(1+z_{e}^{2} x\right) \sinh [\tilde{L} \sqrt{x}]+2 z_{e} \sqrt{x} \cosh [\tilde{L} \sqrt{x}]\right\}} .
$$

This result with $z_{p}=1$ is widely used to analyze experimental data. It reasonably reproduces simulation results of the benchmark $g_{1 Y}(x)$ of Eq. (2.5), for arbitrary scattering anisotropy and boundary reflectivity, as long as the sample is sufficiently thick and the value $z_{e}$ is taken according to Eq. (2.2) [10].

For the same geometry, but for backscattered light, the correlation function is obtained simply by replacing $z_{p}$ by $\widetilde{L}-z_{p}$ in Eq. (2.8). In the semi-infinite limit, $\widetilde{L} \rightarrow \infty$, and for small $x$, this prediction is close to the experimental [12] and simulation $[15,16]$ result of

$$
g_{1 B}(x)=\exp (-\gamma \sqrt{x})
$$


where $\gamma$ is a number close to 2 that depends on boundary reflectivity and scattering anisotropy. This result is widely used to analyze experimental data.

The central predictions of diffusion theory, Eqs. (2.3) and (2.8) with $z_{p}=1$ for DTS and DWS, respectively, rely upon several approximations that are valid only for thick slabs. Besides ignoring any direction dependence in the photon concentration field, they do not account for ballistic propagation or scattering anisotropy, and hence become increasingly inaccurate, and eventually fail altogether, for slabs of decreasing thickness. This is unfortunate because most data are obtained for rather thin slabs, $5<L / l^{*}<20$, where the effects of absorption and laser coherence can be neglected, and where better correlation function statistics can be obtained due to higher count rates and slower decay times.

\section{TELEGRAPHER APPROACH TO TRANSPORT}

This section reviews a recently reported theory of photon transport that we have developed to account for the ballistic nature of photon propagation between successive scattering events [17]. Solutions relevant to diffusing light spectroscopies are developed in the following section, explicitly including the influence of scattering anisotropy.

\section{A. Telegrapher equation}

A complete description of photon transport, neglecting polarization and interference effects, requires that the number density of photons be tracked as a function of time, position, and direction. It is straightforward to develop such a theory of radiative transfer simply by considering all the possible ways for this density to change by the ballistic flow of photons, by the scatter of photons in and out from different directions, and by absorption $[22,18,19]$. However, the resulting Boltzmann equation is difficult to solve, even numerically, and this difficulty ultimately originates in the fact that there is a continuum of directions in three-dimensional space. Diffusion theory, by contrast, is much simpler since it neglects the direction dependence and thus deals only with the total photon density and how it changes by scattering and absorption. As a comparably simple alternative to the full transport theory, we have recently proposed a telegrapher equation for the photon density that improves upon diffusion theory by incorporating ballistic propagation effects for isotropic and anisotropic scattering alike. The physical motivation comes from the exact model of transport in a truly onedimensional space, which can be written down and solved without approximation $[23,16]$. The following telegrapher equation for the total photon density $\varphi(\mathbf{r}, t)$ in three dimensions was proposed by assuming only that it be identical in form to the exact telegrapher equation for one-dimensional transport, but with numerical coefficients guaranteeing the correct ballistic and diffusive limits for three dimensions:

$$
\nabla^{2} \varphi=\frac{\partial^{2} \varphi}{c^{2} \partial t^{2}}+\left(\frac{2}{l_{a}}+\frac{3}{l^{*}}\right) \frac{\partial \varphi}{c \partial t}+\frac{1}{l_{a}}\left(\frac{1}{l_{a}}+\frac{3}{l^{*}}\right) \varphi
$$

where $c$ is the speed of light in the medium, $l^{*}=l_{s} /(1$ $-g$ ) is the photon transport mean free path, $l_{s}$ is the scattering length, $g$ is the average cosine of the scattering angle, and $l_{a}$ is the absorption length. Note that the wave equation with the correct speed $c$ is recovered at short times and that, ignoring absorption, the standard diffusion equation with the correct diffusion coefficient $D=c l^{*} / 3$ is recovered at long times. Furthermore, the treatment of absorption is selfconsistent since $\varphi(\mathbf{r}, t)=\exp \left(-z / l_{a}\right)$ is a solution in the case of negligible scattering, $l^{*} \gg l_{a}$, and since $\varphi(\mathbf{r}, t)=\exp$ $\left(-c t / l_{a}\right)$ is a solution in general. The accuracy of Eq. (3.1) in describing transport in three dimensions was verified by comparison of its Green's function with random-walk simulation results for the spreading of an instantaneous pulse in an infinite three-dimensional medium with varying degrees of scattering anisotropy and absorption [17]. It should be emphasized that Eq. (3.1) describes photon concentration away from a source, independent of the degree of scattering anisotropy; as will be shown in Sec. IV, anisotropy plays an important role only in the treatment of the source.

Since the values of $c$ and $l^{*}$ set the scales for ballistic and diffusive behavior, it is convenient to work in a dimensionless system of units where all lengths are measured in units of $l^{*}$ and all times are measured in units of $l^{*} / c$. The resulting dimensionless telegrapher equation is thus

$$
\nabla^{2} \varphi=\frac{\partial^{2} \varphi}{\partial t^{2}}+\left(2 \mu_{a}+\frac{1}{D_{0}}\right) \frac{\partial \varphi}{\partial t}+\mu_{a}\left(\mu_{a}+\frac{1}{D_{0}}\right) \varphi
$$

where $\mu_{a}=l^{*} / l_{a}$ is the dimensionless absorption coefficient and $D_{0}=1 / 3$ is the dimensionless diffusion coefficient.

\section{B. Boundary conditions and observable flux}

To complement the telegrapher equation for predicting photon transport within and out of a finite medium, it is necessary to specify boundary conditions for $\varphi(\mathbf{r}, t)$ and a prescription for deducing the observable flux of exiting photons. We obtained these [17] again by considering the results of the exact description of the one-dimensional problem. In the same dimensionless system of units as for Eq. (3.2), the result for the boundary conditions is

$$
0=\left.\left[1+\frac{z_{e}}{1+D_{0} \mu_{a}} \hat{n} \cdot \boldsymbol{\nabla}+\frac{D_{0}}{1+D_{0} \mu_{a}} \frac{\partial}{\partial t}\right] \varphi(\mathbf{r}, t)\right|_{\text {boundary }},
$$

where $\hat{n}$ points normal to the boundary away from the medium and where $z_{e}$ is given by the boundary reflectivity according to Eq. (2.2). This is similar to the usual extrapolation length boundary conditions of diffusion theory, except for the $\mu_{a}$ and $\partial / \partial t$ terms that arise from absorption and ballistic transport, respectively. Note, also, that for time-independent problems the extrapolation length decreases with increasing absorption. The result for the exiting flux at some point on the boundary is found similarly as

$$
J(\mathbf{r}, t)=\left.\frac{D_{0}}{z_{e}} \varphi(\mathbf{r}, t)\right|_{\text {boundary }},
$$

which would be identical to Fick's law if the absorption and ballistic terms in the boundary conditions were dropped.

The complete telegrapher theory of three-dimensional photon transport, except for the specification of the source, consists of Eqs. (3.2)-(3.4). It is of comparable simplicity to 
diffusion theory, but is much more accurate at short times and distances and in cases of strong absorption. This was verified by comparison of analytic solutions for a plane source with the results of random-walk simulations for slabs of varying optical thickness and absorption [17]. The telegrapher theory is also superior to the 2-stream theory of Ref. [16] because the latter applies only to geometries that have a one-dimensional symmetry, has the wrong diffusion coefficient, and cannot incorporate the effects of angle-dependent wall reflectivity.

\section{PREDICTIONS}

We now begin presenting new material. In this section, solutions to the telegrapher theory are found for the geometry of greatest experimental interest for diffusing light spectroscopies: a three-dimensional slab with illumination and detection optics arranged such that there is no discrimination against photons according to their lateral motion within the sample. Absorption is not explicitly considered, but can be incorporated easily based on the results presented.

\section{A. Steady plane source}

The solution for the concentration profile when a steady source of strength $\varphi_{0}$ is located at the plane $z_{p} l^{*}$ in from the edge of a nonabsorbing slab of thickness $L=\widetilde{L} l^{*}$ is given by

$$
\varphi(z)=\frac{\varphi_{0}}{D_{0}} \begin{cases}\left(z+z_{e}\right)\left(1-T_{z_{p}}\right), & z<z_{p} \\ \left(\widetilde{L}-z+z_{e}\right) T_{z_{p}}, & z>z_{p}\end{cases}
$$

where the transverse coordinate $z$ is measured in units of $l^{*}$, and $T_{z_{p}}$ is the transmission probability. This obviously satisfies the time-independent telegrapher equation with no absorption, $\nabla^{2} \varphi=0$. It also satisfies the boundary conditions, Eq. (3.3), by vanishing at distance $z_{e} l^{*}$ outside the sample. Furthermore, it correctly gives the diffusely backscattered and transmitted fluxes according to Eq. (3.4) as

$$
\begin{gathered}
\left(D_{0} / z_{e}\right) \varphi(0)=\left(1-T_{z_{p}}\right) \varphi_{0}, \\
\left(D_{0} / z_{e}\right) \varphi(L)=T_{z_{p}} \varphi_{0} .
\end{gathered}
$$

Curiously, however, the value of the transmission probability has not yet been specified even though all the ingredients of the theory have been used. To determine the value of $T_{z_{p}}$ requires physical arguments outside of the standard telegrapher model, just as was required to specify the value of $z_{e}$. To begin, first note that the size of the discontinuity per unit incident flux at the source point, $\Delta \varphi \equiv\left[\varphi\left(z_{p}{ }^{+}\right)\right.$ $\left.-\varphi\left(z_{p}{ }^{-}\right)\right] / \varphi_{0}$, has also not been specified, and that fixing the value of either $T_{z_{p}}$ or $\Delta \varphi$ determines the value of the other. Evaluating the discontinuity at $z_{p}$ according to the profile of Eq. (4.1) and then solving for the transmission probability gives the key result:

$$
T_{z_{p}}=\frac{\left(z_{p}+D_{0} \Delta \varphi\right)+z_{e}}{\widetilde{L}+2 z_{e}} .
$$

Accordingly, the usual diffusion theory prediction for the transmission probability, Eq. (2.3), is recovered by forcing the profile to be continuous at $z_{p}$, while an increase in $\Delta \varphi$ leads to an increase in transmission probability. A nonzero value of the discontinuity can be understood physically as arising from anisotropic scattering. First, if the scattering is anisotropic, then photons first scattered away from an incident beam at $z_{p}$ will be preferentially directed deeper into the sample; therefore, the concentration for $z>z_{p}$ should be greater than for $z<z_{p}$ in proportion to the extent of the anisotropy. Second, the transmission probability should increase as photons begin scattering from deeper within the sample, again in proportion to the extent of the anisotropy. This intuitive connection between the concentration discontinuity at the source point and the extent of scattering anisotropy can also be seen mathematically from the exact 2stream theory of transport in a truly one-dimensional medium. There, Eq. (13) of Ref. [16] shows that the transmission probability has the form of Eq. (4.3) but with $D_{0} \Delta \varphi$ replaced by $2 p_{f}-1$, where $p_{f}$ is the probability for scattering forward without changing streams. For one dimension, the $D_{0} \Delta \varphi$ term appearing in Eq. (4.3) is thus given by the average cosine of the scattering angle, $g=(+1)\left(p_{f}\right)+(-1)(1$ $\left.-p_{f}\right)=2 p_{f}-1$. As shown next, the relation $D_{0} \Delta \varphi=g$ also turns out to be obeyed for thick samples in higher dimensions.

In general, the size of the discontinuity can be deduced in terms of the scattering anisotropy by requiring sensible behavior of the transmission probability in the diffusive and single-scattering limits. For very thick slabs, the total diffuse transmission probability should depend only on the transport mean free path, independent of scattering anisotropy. Averaging $T_{z_{p}}$ over an exponential distribution of penetration depths according to the scattering length gives the total diffuse transmission probability as

$$
\lim _{L \rightarrow \infty} T_{d}=\int_{0}^{\infty} T_{z_{p}} e^{-z_{p} l^{*} / l_{s}} d z_{p} l^{*} / l_{s}=\frac{\left(l_{s} / l^{*}+D_{0} \Delta \varphi\right)+z_{e}}{\widetilde{L}+2 z_{e}}
$$

assuming that the discontinuity is independent of penetration depth. This averaging procedure will be discussed more fully in Sec. IV C. Since the scattering and transport lengths are related by $l_{s} / l^{*}=(1-g)$, the diffuse transmission is independent of anisotropy only if the discontinuity is given by

$$
D_{0} \Delta \varphi=g \text { for } L \gg l^{*} \text {. }
$$

As the scattering anisotropy increases, the average penetration depth decreases and the discontinuity increases in such a way that their sum, $\left(l_{s} / l^{*}+D_{0} \Delta \varphi\right)=1$, remains a constant equal to one. Therefore, the total diffuse transmission probability for thick slabs is simply $T_{d}=\left(1+z_{e}\right) /\left(\widetilde{L}+2 z_{e}\right)$ independent of anisotropy. This solves the old puzzle as to why the ad hoc procedure of setting $z_{p}=1$ in Eq. (2.3) yields good results for any degree of scattering anisotropy.

In the opposite limit of very thin slabs, where photons scatter not more than once, the diffuse transmission probability can be calculated directly and used to deduce the discon- 
tinuity. Summing all the probabilities for a singly scattered photon to reflect any number of times, and then be transmitted, gives

$$
T_{z_{p}}=\int_{0}^{1}\left(\frac{F(\mu)+F(-\mu) R(\mu)}{1+R(\mu)}\right) d \mu
$$

after averaging over forward scattering angles; $F(\mu)$ is the scattering form factor and $R(\mu)$ is the boundary reflectivity. To agree with the $\widetilde{L} \rightarrow 0$ limit of Eq. (4.3), the discontinuity must be taken as

$$
\begin{aligned}
D_{0} \Delta \varphi & =2 z_{e} \int_{0}^{1}\left(\frac{F(\mu)+F(-\mu) R(\mu)}{1+R(\mu)}\right) d \mu-z_{e} \\
& \equiv g^{\prime} \quad \text { for } L \ll l^{*} .
\end{aligned}
$$

If the scattering is isotropic, $F(\mu)=F(-\mu)$, this reduces to zero. If the boundary reflectivity is independent of angle, this reduces to $g^{\prime}=\frac{2}{3}\left(2 p_{f}-1\right)$ where $p_{f}$ is the probability of scattering into the forward direction with a deflection angle of less than $90^{\circ}$. In general, however, it must be evaluated numerically.

In summary, the discontinuity in diffuse photon concentration at the source plane for a three-dimensional slab is fixed by requiring that sensible limits be obtained for very thick and very thin samples. In both the diffusive and single scattering limits, the discontinuity is zero for isotropic scattering and increases as the scattering becomes more aniso- tropic according to Eqs. (4.5) and (4.7). In Sec. V A we will demonstrate explicitly by simulation that a discontinuity in the concentration profile exists and is consistent with these expectations.

\section{B. Instantaneous plane source}

For many problems of interest, including DWS, it is necessary to consider the time-dependent response to an instantaneous pulse. Boundary conditions are most easily implemented using Laplace transform methods. In the telegraphers model, the Laplace transform with respect to time of a pulse of strength $\varphi_{0}$ introduced at depth $z_{p} l^{*}$ into a slab is predicted to have the general form

$$
\bar{\varphi}(z, \omega)=\frac{\varphi_{0}}{2}\left(\frac{\sqrt{\alpha}}{\omega} \pm \Delta \varphi\right) e^{-\sqrt{\alpha}\left|z-z_{p}\right|}+c_{1} e^{\sqrt{\alpha} z}+c_{2} e^{-\sqrt{\alpha} z},
$$

where $\omega$ is the transform variable conjugate to time, $\alpha$ $=\omega\left(\omega+1 / D_{0}\right), z$ is the transverse coordinate, and the plus and minus signs are for $z$ greater and less than $z_{p}$, respectively. As before, lengths are measured in units of $l^{*}$ and times in units $l^{*} / c$. It is straightforward to verify that this satisfies the telegrapher equation, Eq. (3.2), and that the discontinuity per unit source is of size $\Delta \varphi$. The first term represents the source, while the amplitudes $c_{1}$ and $c_{2}$ of the subsidiary terms are chosen in order to satisfy the boundary conditions, Eq. (3.3). Using the prescription of Eq. (3.4) for the exiting flux, the Laplace transform with respect to time of the transmitted pulse is found to be

$$
\frac{D_{0}}{z_{e}} \bar{\varphi}(L, \omega)=\varphi_{0} \frac{\left[1+\left(D_{0}+\Delta \varphi z_{e}\right) \omega\right] \sinh \left[z_{p} \sqrt{\alpha}\right]+\left(z_{e}+D_{0} \Delta \varphi\right) \sqrt{\alpha} \cosh \left[z_{p} \sqrt{\alpha}\right]}{\left[1+\left(z_{e}^{2}+D_{0}^{2}\right) \omega / D_{0}\right] \sinh [\widetilde{L} \sqrt{\alpha}]+2 z_{e} \sqrt{\alpha} \cosh [\widetilde{L} \sqrt{\alpha}]}
$$

The Laplace transform of the backscattered pulse can be found similarly; it can also be obtained from the transmission result simply by replacing $z_{p}$ by $\widetilde{L}-z_{p}$ and $\Delta \varphi$ by $-\Delta \varphi$. As a check, note that the transmission probability is equal to the integral of the transmitted pulse over all time, and is hence given by $\bar{\varphi}(L, 0) D_{0} / z_{e}=\varphi_{0} T_{z_{p}}$. The value thus obtained from the $\omega \rightarrow 0$ limit of Eq. (4.9) is clearly equal to the previous result, Eq. (4.3), found for the steady source. The backscattering probability can be shown, similarly, to be $1-T_{z_{p}}$. This provides an important, nontrivial, check on the internal consistency of the telegrapher equation, boundary conditions, and treatment of the discontinuity at the source. As an aside, note that the influence of absorption on both the pulse shape and transmission probability is found by taking $\omega \rightarrow \omega+\mu_{a}$.

Besides giving transmission probabilities with and without absorption, the Laplace transform results are useful for analysis of other experimental data as well. For instance, they can be inverted for the time dependence of emerging pulses [17]. And, according to Eq. (2.7) for the theory of DWS, they can be evaluated at $\omega=D_{0} x$, and hence $\alpha=x\left(1+D_{0}^{2} x\right)$, then normalized, to approximate the electric field autocorrelation function. For transmission, the result is

$$
g_{1 T, z_{p}}(x)=\frac{\left[1+\left(D_{0}^{2}+g z_{e}\right) x\right] \sinh \left[z_{p} \sqrt{\alpha}\right]+\left(z_{e}+g\right) \sqrt{\alpha} \cosh \left[z_{p} \sqrt{\alpha}\right]}{T_{z_{p}}\left\{\left[1+\left(z_{e}^{2}+D_{0}^{2}\right) x\right] \sinh [\widetilde{L} \sqrt{\alpha}]+2 z_{e} \sqrt{\alpha} \cosh [\widetilde{L} \sqrt{\alpha}]\right\}} .
$$

For backscattering, the result can be generated from this as before. Since the failure of the continuum approximation prevents application to very thin samples, we have set the discontinuity to the thick-sample limit of $D_{0} \Delta \varphi=g$. Note that Eq. (4.10) bears similarity to the diffusion theory prediction, Eq. (2.8), which indeed is recovered by setting both $D_{0}^{2}$ and $g$ to zero. The differences due to the $D_{0}^{2}$ terms indicate the influence of ballistic propagation, while the differences due to the $g$ terms indicate the influence of scattering anisotropy. 


\section{Collimated beam}

The expressions found above for the transmission probability and DWS correlation functions are not quite ready for use in data analysis. First, they must be averaged over a distribution of penetration depths according to the scattering length and wall reflectivity. Considering all multiple reflections of the collimated incident beam from the sample boundaries, the total diffuse transmission probability is found as

$$
\begin{aligned}
T_{d} & =\frac{1}{1-F^{2}} \int_{0}^{\widetilde{L}}\left[T_{z_{p}}+F\left(1-T_{z_{p}}\right)\right] e^{-z_{p} /(1-g)} d z_{p} /(1-g) \\
& =\frac{\left[\left(1-g+D_{0} \Delta \varphi\right)(1-F)+z_{e}(1+F)\right]\left(1-e^{-\widetilde{L} /(1-g)}\right)-\widetilde{L}\left(1-R_{b}\right) e^{-\widetilde{L} /(1-g)}}{\left[\widetilde{L}+2 z_{e}\right]\left(1-F^{2}\right)},
\end{aligned}
$$

where $F \equiv R_{b} e^{-L / l_{s}}=R_{b} e^{-\widetilde{L}(1-g)}$ is the probability for an incident to cross the sample without scattering and then reflect. The $T_{z_{p}}$ and $F\left(1-T_{z_{p}}\right)$ terms in the integral represent the contribution from photons that reflect an even and odd number of times, respectively, before being scattered out of the incident beam at $z_{p}$ (see Fig. 3). The result, Eq. (4.11), is the central new prediction intended for analysis of diffuse transmission data. For thick samples, it reduces to the usual diffusion prediction, Eq. (2.3) with $z_{p}$ set to one. For thinner samples, its behavior and accuracy will be considered in Secs. V and VI; there, we will also recommend an empirical interpolation scheme between the two limits of Eqs. (4.5) and (4.7) for the magnitude of the discontinuity.

Similar averages must be performed for DWS. In backscattering, taking the $L \rightarrow \infty$ limit and then averaging over an exponential distribution of $z_{p}$ according to the scattering length gives

$$
\begin{aligned}
g_{1 B}(x) & =\int_{0}^{\infty} g_{1 B, z_{p}}(x) e^{-z_{p} /(1-g)} d z_{p} /(1-g) \\
& =\frac{1+\left(D_{0}^{2}-g z_{e}\right) x+\left(z_{e}-g\right) \sqrt{\alpha}}{[1+(1-g) \sqrt{\alpha}]\left[1+\left(D_{0}^{2}+z_{e}^{2}\right) x+2 z_{e} \sqrt{\alpha}\right]},
\end{aligned}
$$

where, recall, $x=k^{2}\left\langle\Delta r^{2}(\tau)\right\rangle, \alpha=x\left(1+D_{0}^{2} x\right), D_{0}=1 / 3$, and $g$ is the average cosine of the scattering angle. For DWS in transmission, the result for a plane source must be averaged over $z_{p}$ as well as summed over multiple reflections of the unscattered beam:

$$
g_{1 T}(x)=\frac{\int_{0}^{\widetilde{L}}\left[T_{z_{p}} g_{1 T, z_{p}}(x)+F\left(1-T_{z_{p}}\right) g_{1 B, z_{p}}(x)\right] e^{-z_{p} /(1-g)} d z_{p}}{T_{d}\left(1-F^{2}\right)(1-g)} .
$$

Note that diffusely transmitted photons that remain in the incident beam for an odd number of reflections all contribute according to $g_{1 B, z_{p}}(x)$, since they are traveling away from the transmission boundary when first scattered (see Fig. 3). This integral is straightforward to evaluate, but the answer is rather cumbersome. To obtain a reasonably convenient expression, we set $F=0$ and thus restrict our attention to thicknesses well outside the single-scattering regime. This is not an unnecessarily severe restriction since, by contrast with the diffuse transmission probability, the DWS prediction must fail in the thin slab limit because of the continuum approximation. Evaluating Eq. (4.13) without the multiple reflections and with the discontinuity set to $D_{0} \Delta \varphi=g$ then gives the central prediction intended for use in analysis of actual data:

$$
g_{1 T}(x)=\frac{(A C+B)-\{(A+B C) \sinh [\tilde{L} \sqrt{\alpha}]+(A C+B) \cos [\tilde{L} \sqrt{\alpha}]\} \exp [-\tilde{L} /(1-g)]}{T_{d}^{\prime}\left(1-C^{2}\right)\left\{\left[1+\left(z_{e}^{2}+D_{0}^{2}\right) x\right] \sinh [\tilde{L} \sqrt{\alpha}]+2 z_{e} \sqrt{\alpha} \cosh [\tilde{L} \sqrt{\alpha}]\right\}},
$$

where the coefficients are defined as

$$
\begin{gathered}
A=1+\left(D_{0}^{2}+g z_{e}\right) x, \\
B=\left(z_{e}+g\right) \sqrt{\alpha}, \\
C=(1-g) \sqrt{\alpha},
\end{gathered}
$$

$$
T_{d}^{\prime}=\frac{\left(1+z_{e}\right)-\left(1+z_{e}+\widetilde{L}\right) \exp [-\widetilde{L} /(1-g)]}{\widetilde{L}+2 z_{e}} .
$$

This result includes the effects of boundary reflectivity through $z_{e}$, scattering anisotropy through $g=1-\left(l_{s} / l^{*}\right)$, and ballistic propagation through $D_{0}^{2}$, all of which are important for typical slabs of thickness $L<15 l^{*}$. For thick slabs, it reduces to the usual diffusion theory prediction, Eq. (2.8) 


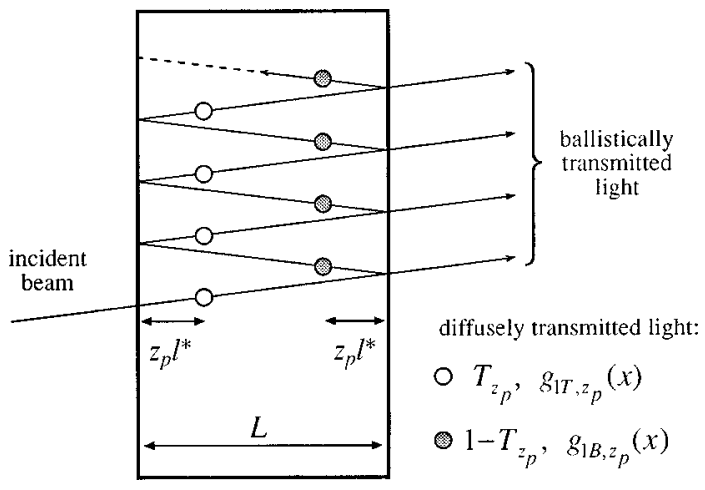

FIG. 3. Diffusing photons are created by scattering away from the incident beam. The complete source is thus constructed by integrating the plane-source results over the penetration depth, $z_{p}$, and summing over all multiple reflections.

with $z_{p}$ set to one. The effects of absorption on both the transmission probability and the DWS signal can be obtained by taking $x \rightarrow x+\mu_{a} / D_{0}$ and then normalizing.

To examine the influence of anisotropic scattering and ballistic propagation, we investigate the approach of the prediction, Eq. (4.14), to the thick-sample limit $g_{1}(x)$ $=\widetilde{L} \sqrt{x} / \sinh (\widetilde{L} \sqrt{x})$. We thus define an expansion by $\ln \left[g_{1}(x)\right]=-\left(\Gamma_{1} x\right)+\frac{1}{5} A\left(\Gamma_{1} x\right)^{2}+O\left(x^{3}\right)$, where $\Gamma_{1}$ is the first cumulant controlling the decay rate, and $A$ is a shape parameter specifying the initial curvature on a semilogarithmic plot. Straightforward manipulation gives

$$
\begin{aligned}
\Gamma_{1}= & \frac{\widetilde{L}^{2}}{6}\left[\frac{1+6 z_{e} \widetilde{L}^{-1}-6\left(1-z_{e}^{2}-D_{0}^{2}\right) L^{-2}-12 z_{e} \widetilde{L}^{-3}}{1+2 z_{e} \widetilde{L}^{-1}}\right] \\
& +\frac{g\left(2+z_{e}-g+D_{0}^{2}\right)-D_{0}^{2}}{1+z_{e}}+O\left(\widetilde{L^{3}} \exp [-\widetilde{L} /(1-g)]\right), \\
A= & 1+12\left[\left(1+z_{e}^{2}\right)-\frac{g\left(2+z_{e}-g+D_{0}^{2}\right)+D_{0}^{2}\left(5+7 z_{e}\right) / 2}{1+z_{e}}\right] \\
& \times \widetilde{L}^{-2}+O\left(\widetilde{L}^{-3}\right) .
\end{aligned}
$$

The leading thick-slab behaviors are $\Gamma_{1} \cong \widetilde{L}^{2} / 6$, since transmitted photons typically take this number of completely random steps of size $l^{*}$ in crossing a slab, and $A \cong 1$, by definition. According to Eq. (4.16), the first correction to the cumulant scales as $\widetilde{L}^{-1}$ and depends only on boundary reflectivity; anisotropy and ballistic effects are relatively unimportant even for rather thin slabs. The leading correction to the shape, by contrast, scales as $\widetilde{L}^{-2}$ and depends crucially on all these quantities. In particular, the degree of upward curvature increases for larger $z_{e}$ and for smaller $g$. Physically, according to the fundamental DWS equation (2.7), this can be understood in terms of an increase in the width of the photon path length distribution $P(S)$. If the scattering is highly anisotropic, then all photons scatter out of the ballistic incident beam very close to the boundary and hence begin diffusing at the same location. If the scattering is isotropic, by contrast, then photons scatter out of the incident beam over a broader range of distances and this leads to a broader distribution of diffusive path lengths. The influence of bal- listic transport can be similarly understood. The telegrapher theory obeys causality by forcing $P(S)=0$ for $S<\widetilde{L}-z_{p}$ [17]. The standard diffusion theory, recovered by taking $D_{0}^{2}$ $=0$, does not exclude these short paths and hence gives a broader distribution of diffusive path lengths and more upward curvature.

\section{SIMULATIONS}

The remainder of the paper presents various tests of the above predictions, in this section by random-walk simulation and in the next by experiment. Here, random walkers are launched in from the edge of a slab and allowed to wander until exiting from either the transmission or backscattering sides. Following the methods of Refs. [9,10], steps are generated with an exponential distribution of sizes according to the scattering length, $l_{s}$, and a distribution of directions given by the Henyey-Greenstein scattering form factor, $F(\mu)=\frac{1}{2}\left(1-g^{2}\right)\left(1+g^{2}-2 \mu g\right)^{-3 / 2}$, where $g$ $=\int_{-1}^{1} \mu F(\mu) d \mu$ is the average cosine of the scattering angle [24]. When a walker encounters the boundary at either edge of the slab, it either exits or is reflected specularly back into the sample according to an angle-independent reflection probability, $R$. Isotropic and anisotropic random walks generated by this procedure are compared in Fig. 1.

\section{A. Concentration profile}

The treatment of scattering anisotropy by a discontinuity in photon concentration at the source point is the key theoretical idea in this paper, and the size of the discontinuity, Eq. (4.5) or (4.7), is a crucial input to DTS and DWS predictions. Therefore, we first use random-walk simulations to study the existence and magnitude of the discontinuity in relation to scattering anisotropy. We proceed by collecting statistics for the diffuse transmission probability and the profile of photon concentration versus distance. To mimic scattering away from a normally incident beam at a distance $z_{p} l^{*}$ in from the edge of the sample, as in the predictions to be tested, the very first step starts at $z_{p} l^{*}$ and is directed away from the $+z$ direction according to the form factor. Altogether, then, four inputs must be specified: the value of the slab thickness, $L / l^{*}$, the boundary reflectivity $R$, the scattering anisotropy $g$, and the penetration depth ratio $z_{p}$. For each choice, results for the transmission probability $T_{z_{p}}$ are obtained by tallying the number of transmitted walkers. Results for the steady, time-averaged concentration profile are obtained by binning up the amount of time each walker spends at a given depth in the sample. Example data are shown in Fig. 2 for a penetration depth of $z_{p}=1$ into slabs of thickness $L / l^{*}=10$ for six different combinations of scattering anisotropy, $g \in\{0,0.5,0.9\}$, and boundary reflectivity, $R \in\{0,1 / 2\}$. For comparison, the prediction of Eq. (4.1) is also plotted in Fig. 2, where $T_{z_{p}}$ is taken from the simulation value, $z_{e}$ is taken from Eq. (2.2), and $\varphi_{0}$ is the total time spent by all walkers inside the slab; there are no free parameters in the comparison. In all cases, the predicted and simulated profiles agree very well throughout the entire slab, except very close to the source. In particular, the profiles are all nearly linear on each side of the source plane and extrapolate to zero at $z_{e}=2 / 3$ and 2 outside the sample for $R=0$ and $1 / 2$, 


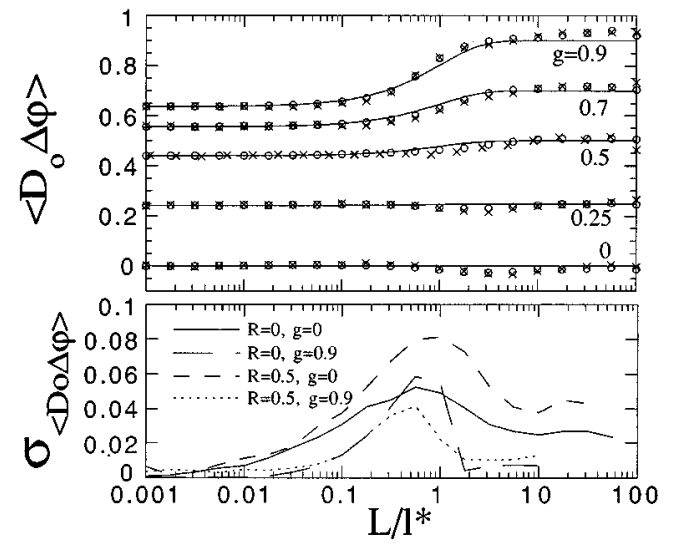

FIG. 4. Average size and rms deviation of the discontinuity, as defined by the weighting over $z_{p}$ in Eq. (5.1) vs slab thickness for several degrees of anisotropy as labeled. Results are seen to be independent of boundary reflectivity, open circles for $R=0$ and crosses for $R=1 / 2$, and to agree well with the limiting values of Eqs. (4.5) and (4.7); the solid curves represent the empirical interpolation of Eq. (5.2).

respectively. But more importantly, it also shows how the profiles are not necessarily continuous at $z_{p}$. The size of the discontinuity can be read straight off the plots to be $D_{0} \Delta \varphi$ $\cong 0,0.5$, and 0.9 for the three cases $g=0,0.5$, and 0.9 , respectively, independent of wall reflectivity. This provides strong confirmation of the expectation $D_{0} \Delta \varphi=g$ of Eq. (4.5) for thick samples.

Next, the behavior of the discontinuity at the source is examined as a function of slab thickness. It is also important to examine the behavior of the discontinuity as a function of $z_{p}$, since in a real experiment diffusing photons are introduced over an exponential range of penetration depths as photons are scattered out of the incident beam. Thus Fig. 4 displays statistics for the average and rms deviation of the discontinuity as a function of slab thickness, for two different values of wall reflectivity and several degrees of scattering anisotropy. The averages shown are defined as follows by exponential weighting according to the scattering length, as would occur in experiment:

$$
\left\langle D_{0} \Delta \varphi\right\rangle=\frac{\int_{0}^{L / l^{*}}\left(D_{0} \Delta \varphi_{z_{p}}\right) e^{-z_{p} l^{*} / l_{s}} d z_{p} l^{* / l_{s}}}{\int_{0}^{L / l^{*}} e^{-z_{p} l^{*} / l_{s}} d z_{p} l^{*} / l_{s}},
$$

where $D_{0} \Delta \varphi_{z_{p}}$ is the discontinuity at $z_{p}$ as deduced from the simulation result for $T_{z_{p}}$ using Eq. (4.3). The simulation results for the rms deviation, defined similarly as $\sigma_{\left\langle D_{0} \Delta \varphi\right\rangle}$ $=\sqrt{\left\langle\left(D_{0} \Delta \varphi\right)^{2}\right\rangle-\left\langle D_{0} \Delta \varphi\right\rangle^{2}}$, show that the discontinuity is independent of $z_{p}$ to a good approximation, more so for stronger anisotropy. So it is enough to consider the average discontinuity as a function of slab thickness and anisotropy, without regard to $z_{p}$. The results for $\left\langle D_{0} \Delta \varphi\right\rangle$ versus $L / l^{*}$ displayed in the top plot of Fig. 4 also conform very well to the expectations of Eqs. (4.5) and (4.7) independent of wall reflectivity. This is illustrated by close agreement of the

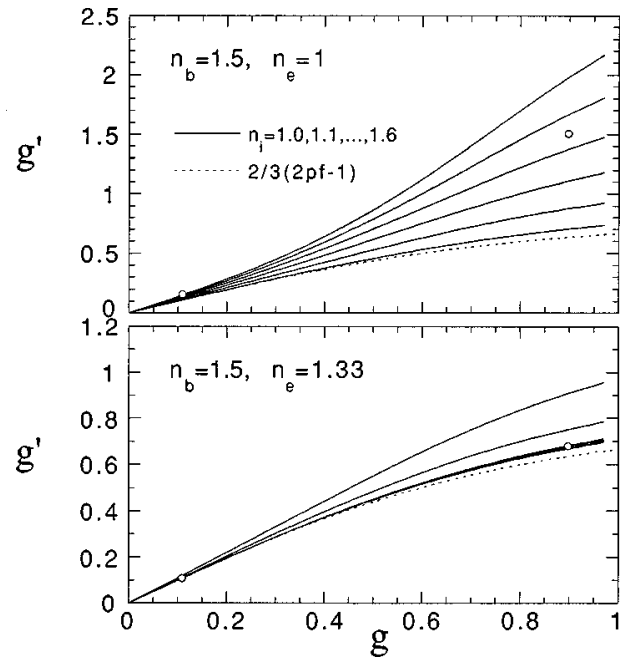

FIG. 5. Required discontinuity in diffuse-photon concentration in the single-scattering limit, Eq. (4.7), vs scattering anisotropy for samples held on glass cells and measured in air (top) or water (bottom). In both cases, the dashed curve is the constant reflectivity limit and the solid curves are for interior indices of 1.0 through 1.6 from bottom to top. The open points represent values for the isotropic and anisotropic samples described in Sec. VI.

simulation data with the solid curves that interpolate exponentially between the expected small and thick slab limits according to

$$
\left\langle D_{0} \Delta \varphi\right\rangle=g+\left(g^{\prime}-g\right) \exp \left(-L / l^{*}\right) .
$$

This empirical relationship works well for all reflectivities and anisotropies examined. As long as the slab thickness is greater than $L=2 l^{*}$, the discontinuity can therefore be taken as $\left\langle D_{0} \Delta \varphi\right\rangle=g$. And as long as the thickness is less than $L$ $=0.1 l^{*}$, the discontinuity can be taken as $\left\langle D_{0} \Delta \varphi\right\rangle=g^{\prime}$. It is curious that the thin slab limit, and the crossover between the two regimes, is set by the value of $l^{*}$ rather than by the scattering length.

In the cases of constant reflectivity simulated above, the discontinuity in the single scattering regime is simply $g^{\prime}$ $=\frac{2}{3}\left(2 p_{f}-1\right)$. In many experiments, the scattering medium is a liquid suspension held in a glass cell and measured in air or an index-matching bath. In these cases, the discontinuity must be calculated numerically using the angle-dependent Fresnel reflectivity of both interior-wall and wall-exterior interfaces. Results for a variety of liquid refractive indices are shown in Fig. 5 for a glass index of 1.5 and the HenyeyGreenstein form factor. For any set of refractive indices at the boundary, note that the discontinuity approaches the constant-reflectivity value $g^{\prime} \rightarrow \frac{2}{3}\left(2 p_{f}-1\right)=g-O\left(g^{3}\right)$ for small $g$. Thus, for weak anisotropy, $g<0.4$, excellent results should be obtained by treating the discontinuity as a constant independent of thickness; this can be seen in Fig. 4.

\section{B. Diffuse transmission}

With the foundation now established, we may begin testing the predictions for diffusing-light spectroscopies. The only difference in simulation procedure is that now the location of the first scattering event away from the incident beam is not specified, but is rather taken at random for each walker 


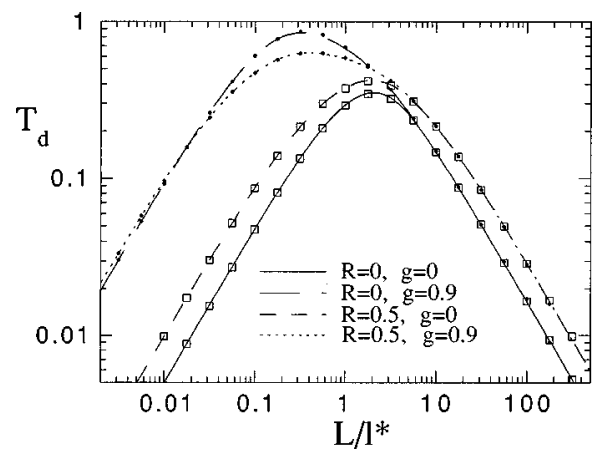

FIG. 6. Total diffuse transmission vs slab thickness for four combinations of boundary reflectivity and scattering anisotropy, as labeled. Symbols represent results of random-walk simulations and curves represent the prediction of Eq. (4.11) using the average discontinuity given by Eq. (5.2).

according to the scattering length and wall reflectivity. Results for the total diffuse transmission probability as a function of optical thickness are shown as symbols in Fig. 6 for four different combinations of boundary reflectivity, $R$ $\in\{0,1 / 2\}$, and scattering anisotropy, $g \in\{0,0.9\}$; the corresponding predictions from Eq. (4.11) are shown as curves. In the single scattering regime, $L \ll l_{s}$, the diffuse transmission increases linearly with thickness and depends on both the reflectivity and the anisotropy. Throughout this regime, the simulation data and the telegrapher predictions are in perfect agreement, as expected by the choice of discontinuity. In the diffuse regime, $L \gg l^{*}$, the transmission decreases with thickness in proportion to $L^{-1}$ and depends strongly on reflectivity, but not on anisotropy. This is seen in both the simulation data and in the predictions of Eq. (4.11), which are indistinguishable to within about $\pm 1 \%$ for $L / l^{*} \geqslant 2$. The deviation of simulation from prediction is greatest in between the two limiting regimes, but, as seen in Fig. 6, is never more than a few percent. Thus, by properly including boundary reflectivity through an extrapolation length and scattering anisotropy through a discontinuity at a source that is exponentially distributed according to the scattering length, the transmission probability can be predicted from one simple theory with great accuracy in both the single- and multiple-scattering limits as well as in the difficult region in between.

\section{Diffusing-wave spectroscopy}

The accuracy of the DWS correlation function predictions, Eq. (4.12) for backscattering and Eq. (4.14) for transmission, can be also be gauged by comparison with randomwalk simulations. As walkers wander through the slab, both their total square wave-vector transfer and their total path length are measured, and the results are used respectively to compute the correlation function according to the benchmark, $g_{1 Y}(x)$ of Eq. (2.5), and the continuum approximation, $g_{1 S}(x)$ of Eq. (2.7).

\section{Backscattering}

Simulation results of the DWS correlation function in the backscattering geometry are shown in Fig. 7 for four different combinations of boundary reflectivity, $R \in\{0,1 / 2\}$, and scattering anisotropy, $g \in\{0,0.9\}$, along with the telegrapher prediction of Eq. (4.12). As observed previously $[15,10]$, the

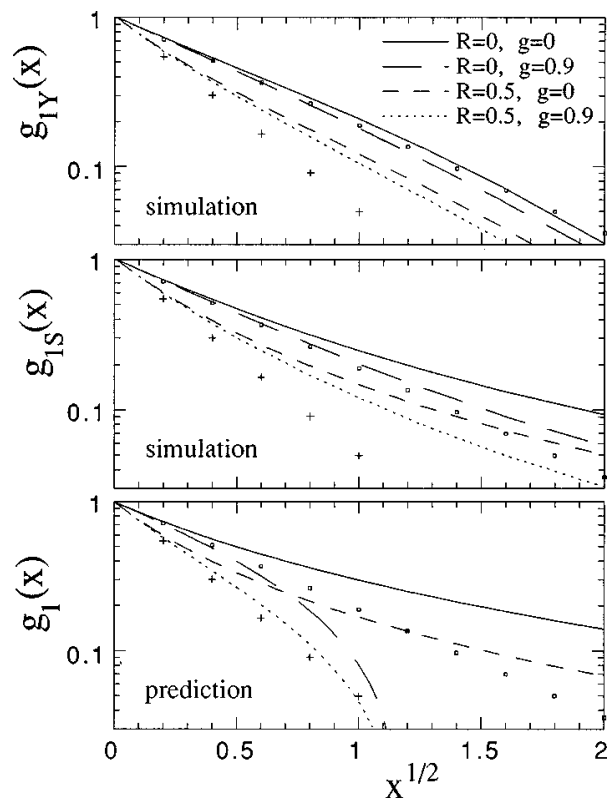

FIG. 7. Normalized electric field correlation function for backscattering from a semi-infinite slab for four combinations of boundary reflectivity and scattering anisotropy, as labeled. The top plot shows simulation results for the benchmark, $g_{1 Y}(x)$ of Eq. (2.5), based on momentum transfer; the middle plot shows simulation results for $g_{1 S}(x)$ of Eq. (2.7), based on path length; and the bottom plot shows the telegrapher predictions of Eq. (4.12). The empirical result $g_{1}(x)=\exp \left[-\left(1+z_{e}\right) \sqrt{x}\right]$, based on the small $x$ expansion in Eq. (5.3), is shown in all plots by symbols, open squares for $R$ $=0$ and plusses for $R=1 / 2$, independent of scattering anisotropy.

benchmark $g_{1 Y}(x)$ and the approximation $g_{1 S}(x)$ agree very well for small $x$ (early times), where the signal is dominated by photons with long paths, but disagree for large $x$ (late times), where the signal is dominated by photons that have short paths. This is because the continuum approximation is valid only for long paths consisting of many scattering events. For small $x$, where $g_{1 Y}(x)$ and $g_{1 S}(x)$ are in agreement, the telegrapher prediction is also quite accurate. Namely, the initial decay is nearly exponential in $\sqrt{x}$ and depends on reflectivity but not on anisotropy, while for slightly larger $x$ the decay rate increases slightly with increasing $g$. This can be seen both in Fig. 7 and in the small$x$ expansion of the backscattering prediction, Eq. (4.12):

$$
g_{1}(x)=1-\left(1+z_{e}\right) \sqrt{x}+\left(1-g+z_{e}+z_{e}^{2}\right) x+O\left(x^{3 / 2}\right) .
$$

For even larger $x$, beyond about $x=1$ where $g_{1 Y}(x)$ has decayed to about 0.2 , the continuum approximation fails altogether since, as seen in Fig. 7, there is no longer any quantitative similarity of $g_{1 S}(x)$ to $g_{1 Y}(x)$. In this regime, neither diffusion theory nor telegrapher theory approximations of $g_{1 S}(x)$ can hope to capture the true behavior of $g_{1 Y}(x)$; and indeed, the telegrapher predictions shown in Fig. 7 break down, especially for strong anisotropy. In short, there are as yet no truly satisfactory predictions for the backscattering correlation function based on Eq. (2.7) for $g_{1 S}(x)$, since the continuum approximation breaks down for short paths and since short paths dominate the signal for $g_{1}(x)<0.2$. The best means of analyzing data may still be the empirical form 


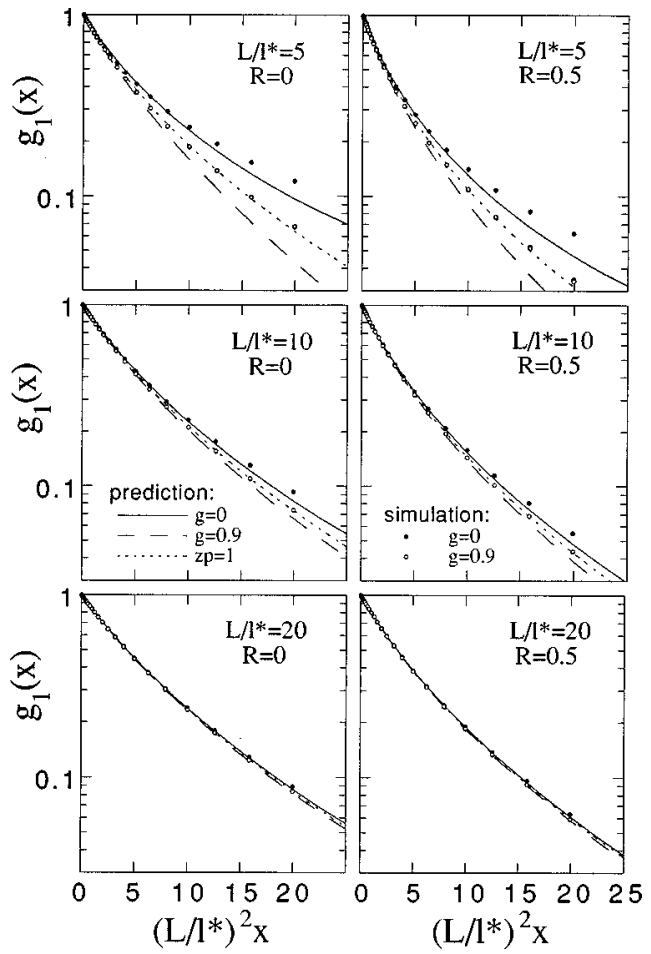

FIG. 8. Normalized electric field correlation function for transmission through slabs of various optical thicknesses and boundary reflectivities, as labeled. Simulation results for the benchmark, $g_{1 Y}(x)$ of Eq. (2.5), based on momentum transfer, are shown as symbols. The telegrapher prediction of Eq. (4.14) is shown by solid and dashed curves. The diffusion theory prediction of Eq. (2.8), which does not distinguish between different levels of scattering anisotropy, is shown by dotted curves.

$g_{1}(x)=\exp (-\gamma \sqrt{x})$ where $\gamma$ is an unknown, adjustable parameter close to $1+z_{e}$. This form is shown by symbols in Fig. 7 for the two cases $\gamma=5 / 3$ and 3, corresponding through the first term in Eq. (5.3) to $z_{e}=2 / 3$ and 2, respectively. This, of course, fails to capture the anisotropy dependence of the decay; it also does not provide good agreement with $g_{1 Y}(x)$ beyond about $x=1$. Since the second term in the expansion in Eq. (5.3) seems reliable, it may be possible to concoct an even better empirical form using this as a guide.

\section{Transmission}

In the transmission geometry, there is a well-defined typical photon path consisting of roughly $\left(L / l^{*}\right)^{2}$ completely random steps of average size $l^{*}$. This implies that the decay of the correlation function is nearly exponential in $\left(L / l^{*}\right)^{2} x$. It also implies that failure of the continuum approximation is a concern only for thin slabs, and can never be as severe as in backscattering. As found previously [10] the difference between the average dimensionless path length $\langle S\rangle$ and the average dimensionless momentum transfer $\langle Y\rangle$, whose values determine the initial decay rates of $g_{1 S}(x)$ and $g_{1 Y}(x)$, respectively, vanishes for thick slabs and is never greater than about $7 \%$ even for slabs as thin as $L=2 l^{*}$. Therefore, the prediction of Eq. (4.14) is tested in Fig. 8 by comparison with $g_{1 Y}(x)$, only, as a function of $\left(L / l^{*}\right)^{2} x$. Simulation results and telegrapher predictions for the transmission correlation function are displayed for three different slab thick-

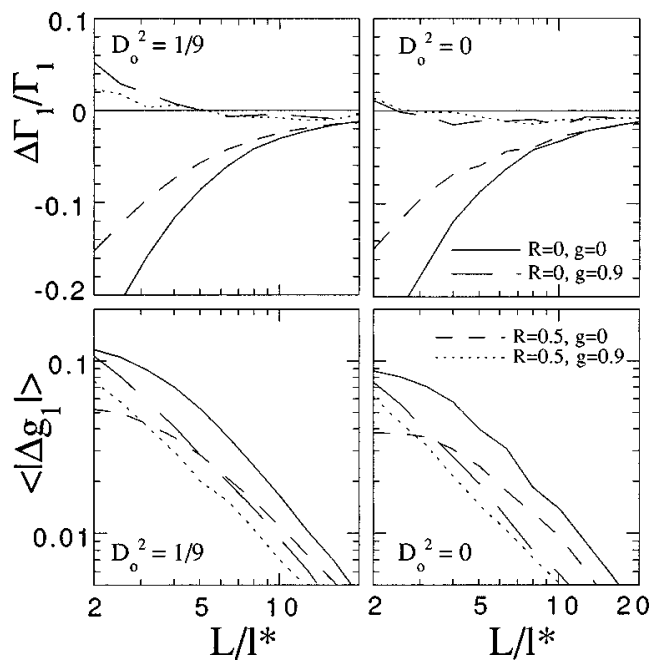

FIG. 9. Quantitative differences between the telegrapher prediction of $g_{1}(x)$ in transmission, Eq. (4.14), and the benchmark transmission correlation function, $g_{1 Y}(x)$ of Eq. (2.5), vs slab thickness. The top plots show the fractional cumulant difference, and the bottom plots show the average absolute shape deviation, Eq. (5.4). Boundary reflectivity and scattering anisotropy are labeled. The left two plots are for the full telegrapher prediction, while the right plots with $D_{0}^{2}=0$ show that better agreement can be obtained by neglecting ballistic propagation effects.

nesses, $L / l^{*}=5,10$, and 20 , with four different combinations of boundary reflectivity, $R \in\{0,1 / 2\}$, and scattering anisotropy, $g \in\{0,0.9\}$. For small $\left(L / l^{*}\right)^{2} x$, the initial decay is nearly exponential in $\left(L / l^{*}\right)^{2} x$ with a rate that depends significantly on reflectivity, but not much on anisotropy, such that the sensitivity to a change in reflectivity increases for thinner slabs. This can be seen both by careful inspection of the figure and of the small- $x$ cumulant expansion of the transmission prediction in Eq. (4.16). For larger $\left(L / l^{*}\right)^{2} x$, the correlation functions all exhibit upward curvature by an amount that depends on thickness, reflectivity, and anisotropy, such that the sensitivity to a change in anisotropy increases for thinner slabs. For very thick slabs, $L \gg l^{*}$, the behavior reduces to the limiting form $g_{1}(x)$ $=\widetilde{L} \sqrt{x} / \sinh (\widetilde{L} \sqrt{x})$ independent of reflectivity and anisotropy. For slabs of intermediate thickness, $20<L / l^{*}<100$, the behavior depends noticeably on reflectivity but not on anisotropy. For thinner slabs, $L / l^{*}<15$, where most experiments are performed, the behavior depends significantly on both reflectivity and anisotropy. As evident in Fig. 8, the telegrapher prediction of Eq. (4.14) captures this entire range of behavior and is quantitatively accurate to a remarkably high degree. By contrast, the diffusion theory prediction of Eq. (2.8), also shown in Fig. 8, fails to distinguish the crucial differences due to scattering anisotropy, and thus becomes only qualitatively correct for thinner slabs. Curiously, as noticed earlier in Ref. [8], it works best for strong anisotropy, the very case for which its transport approximations are most inaccurate.

The differences between the simulated and predicted correlation functions observed in Fig. 8 are difficult to fully quantify. Two reasonable means are shown in Fig. 9 that may be useful to experimentalists for knowing and minimizing the systematic error introduced by analysis of data with 
the telegrapher prediction. The top plot shows the fractional cumulant difference, $\Delta \Gamma_{1} / \Gamma_{1} \equiv\left(\Gamma_{1}-\Gamma_{1 Y}\right) / \Gamma_{1 Y}$, between the initial decay rates of Eq. (4.14) and the benchmark, $g_{1 Y}(x)$, for the usual four combinations of boundary reflectivity and scattering anisotropy. The differences all decrease with increasing thickness, as expected, and even in the worst cases are less than $10 \%$ for thicknesses greater than $L$ $=4 l^{*}$. In the case of moderate reflectivity and anisotropy, as in a typical experiment, the difference is less than a few percent even in slabs as thin as $L=2 l^{*}$. While the cumulant difference serves to quantify the accuracy of the decay rate, the following absolute difference serves to quantify the accuracy of the shape:

$$
\left\langle\left|\Delta g_{1}\right|\right\rangle \equiv \int\left|g_{1}\left(x \Gamma_{1 Y} / \Gamma_{1}\right)-g_{1 Y}(x)\right| d \ln x / \ln 10 .
$$

Since the predicted and benchmark correlations functions both decay nearly exponentially from one to zero, their difference is greatest over about one decade in $x$; therefore, Eq. (5.4) is an indicator of the average difference [10]. Furthermore, since the decay rates have been matched, this quantity solely reflects differences in shape. Simulation results for Eq. (5.4) are shown in the bottom plot of Fig. 9. As expected, all decrease with thickness, and even in the worst cases are below 0.1 for thickness greater than $L=3 l^{*}$. Since the error in the prediction becomes comparable to the benchmark when the correlation function decays below $\left\langle\left|\Delta g_{1}\right|\right\rangle$, a simple rule for avoiding undue systematic error would be to restrict analysis to the portion of the decay satisfying $g_{1}(\tau)$ $>\left\langle\left|\Delta g_{1}\right|\right\rangle$. Since it is routine to measure $g_{1}(\tau)$ down to 0.03, the results in Fig. 9 show that even in the worst case no data need be discarded if the thickness is greater than about $L=7 l^{*}$. For moderate reflectivity and anisotropy, as in a typical experiment, no data need be discarded if the thickness is greater than about $L=4 l^{*}$, and more than a full decade of decay is available even for slabs as thin as $L$ $=2 l^{*}$.

\section{EXPERIMENTS}

While the above random-walk simulations are useful for cleanly isolating and testing the influence of scattering anisotropy, they fail to account for potentially important phenomena that occur in real experiments. This includes the field properties of light, i.e., polarization and interference, as well as angle-dependent behavior in the boundary reflectivity and scattering form factor that are not accounted for by the average quantities $z_{e}$ and $g$, respectively. Therefore, we have performed a series of diffuse transmission and DWS experiments on colloidal suspensions of polystyrene spheres (polyballs). The heart of our approach is to design two suspensions such that, except for scattering anisotropy, their optical and dynamical properties are as much alike as possible.

\section{A. Sample design}

Polystyrene spheres were chosen because the details of their light scattering behavior and their thermal motion are both well known, and can thus be suitably tailored. For the low concentrations employed here, the former are given by
Mie theory in terms of the dielectric constants of the materials and the ratio of the wavelength of light to sphere size $[25,26]$. We use stock polystyrene spheres with diameters of 93 and $653 \mathrm{~nm}$, each with less than $10 \%$ polydispersity (Duke Scientific, Palo Alto CA), and coherent light from an $\mathrm{Ar}^{+}$laser with a wavelength of $\lambda=514.5 \mathrm{~nm}$ in air. Since the 93-nm spheres are much smaller than the wavelength of light, they scatter photons with approximately equal probability into the forward and backward directions according to a simple Rayleigh form factor. Even though the scattering is polarization dependent, especially near $90^{\circ}$, we will refer to these as our "isotropic" samples. By contrast, since the 653 $\mathrm{nm}$ spheres are larger than the wavelength of light, they scatter photons preferentially into the forward direction according to a complicated Mie scattering form factor, we call these our "anisotropic" samples. In both cases, the important length scales are the scattering and transport mean free paths, given, respectively, by

$$
l_{s}=\frac{1}{\rho_{n} \sigma} \quad \text { and } \quad l^{*}=\frac{l_{s}}{1-g},
$$

where $\rho_{N}$ is the number density of spheres, $\sigma$ is the scattering cross section, and, as usual, $g$ is the average cosine of the scattering angle that parametrizes the degree of scattering anisotropy.

The thermal Brownian motion of the spheres in suspension is approximately diffusive, so the dynamical variable appearing in DWS predictions is

$$
x=k^{2}\left\langle\Delta r^{2}(\tau)\right\rangle \cong 6 \tau / \tau_{0} \quad \text { with } \quad \tau_{0}=1 / D k^{2},
$$

where $k$ is the wave vector of light in the medium, $\left\langle\Delta r^{2}(\tau)\right\rangle$ is the average mean-squared displacement of the spheres in time $\tau, D$ is the sphere self-diffusion coefficient, and $\tau_{0}$ is the characteristic time scale required for the spheres to diffuse across one wavelength. Because of hydrodynamic interactions that depend on the volume fraction $\phi$ occupied by spheres, the diffusion coefficient is smaller than the StokesEinstein value according to $D=D_{0}(1-1.83 \phi)$ [27-29]. And because of the hydrodynamic self-interaction, there is a long-time tail in the decay of the velocity autocorrelation function. The detailed functional form of $x(\tau)$ is found [30] to be given by Hinch's prediction $[31,8]$ with the above diffusion coefficient and with a self-interaction time of $\tau_{\nu}=(1$ $-2.5 \phi) a^{2} \rho / \eta$ where $a$ is the sphere radius, $\rho$ is the liquid density, and $\eta$ is the liquid viscosity. The limit of truly diffusion motion described by $\tau_{0}$ in Eq. (6.2) is attained only for $\tau \gg \tau_{\nu}$.

With these well-established ingredients, we may now engineer our samples. First, stock solutions of the $653 \mathrm{~nm}$ spheres are diluted with sufficient water that the transport mean free path is predicted to have a convenient value of $l^{*}=0.52 \mathrm{~mm}$. As recorded in Table I, this produces samples with a large anisotropy parameter of $g=0.90$ and a characteristic diffusion time of $\tau_{0}=5.7 \mathrm{~ms}$. Next, stock solutions of the $93 \mathrm{~nm}$ spheres are mixed with carefully chosen volumes of water and glycerol so that the resulting suspensions are predicted to have the same values of both $l^{*}$ and $\tau_{0}$. This produces samples with a small anisotropy parameter of $g$ $=0.11$, but with optical and dynamical properties that are 
TABLE I. Sample characteristics at a temperature of $21{ }^{\circ} \mathrm{C}$ for light at an incident wavelength of $514.5 \mathrm{~nm}$ in air. The polyball and glycerol fractions are chosen so that the values of $l^{*}$ and $\tau_{0}$ are the same for both the isotropic and anisotropic samples. The manufacturer's reported error in the polyball diameter is $\pm 1 \mathrm{~nm}$; the reported polydispersities are much larger, as quoted below.

\begin{tabular}{lcc}
\hline \hline \multicolumn{1}{c}{ Designed } & Isotropic & Anisotropic \\
\hline Polyball diameter $(\mathrm{nm})$ & $93 \pm 8$ & $653 \pm 12$ \\
Polyball volume fraction $(\%)$ & $1.866 \pm 0.002$ & $0.4134 \pm 0.0004$ \\
Polyball refractive index & $1.60 \pm 0.01$ & $1.60 \pm 0.01$ \\
Glycerol mass fraction $(\%)$ & $53.0 \pm 0.1$ & \\
Liquid refractive index & $1.407 \pm 0.003$ & $1.334 \pm 0.001$ \\
Liquid viscosity $(\mathrm{g} / \mathrm{cm}-\mathrm{s})$ & $0.0752 \pm 0.0005$ & $0.0099 \pm 0.0003$ \\
Liquid density $(\mathrm{g} / \mathrm{cm} 3)$ & $1.135 \pm 0.003$ & $0.998 \pm 0.003$ \\
\multicolumn{1}{c}{$\quad$ Calculated } & & \\
\hline$g$ & $0.11 \pm 0.02$ & $0.898 \pm 0.004$ \\
$l^{*}$ (mm) & $0.52 \pm 0.17$ & $0.52 \pm 0.05$ \\
$\tau_{0}(\mathrm{~ms})$ & $5.7 \pm 0.5$ & $5.7 \pm 0.3$ \\
$\tau_{\nu}(\mathrm{ns})$ & $0.31 \pm 0.06$ & $107 \pm 8$ \\
$z_{e}$ for glass cell in air bath & $2.03 \pm 0.05$ & $1.76 \pm 0.02$ \\
$z_{e}$ for glass cell in water bath & $0.81 \pm 0.01$ & $0.71 \pm 0.01$ \\
\hline \hline
\end{tabular}

otherwise very similar to the anisotropic samples. While the two samples have the same $l^{*}$, note that their scattering lengths $l_{s}=(1-g) l^{*}$ differ by a factor of ten.

After mixing, the suspensions are sealed into two sets of sample cells. The first set consists 10 borosilicate cells (Corning 7740, $n=1.474$ ) with rectangular cross section and with nominal thicknesses ranging from 1 to $10 \mathrm{~mm}$. These were made by extrusion and the actual thickness was taken as the average found along the cell width. The second set consists of 5 cylindrical spectrophotometer-type cells (NSG Precision Cells, Farmingdale, NY) with accurately constructed thicknesses of $0.1,0.2,0.5,1$, and $2 \mathrm{~mm}$. In all cases, the width of the cells is sufficiently great that essentially no photons escape from the sides.

Because of uncertainties in the concentrations and in the liquid and sphere properties, the values of $l^{*}$ and $\tau_{0}$ may not be precisely matched for our actual isotropic and anisotropic samples. Reasonable estimates for the errors are given in Table I, and were obtained as follows. First, the polyball diameters are reported by the manufacturer, and their refractive indices are taken from the literature [32]. The mass and volume fractions are obtained by weighing. As a check, the liquid refractive indices are measured with an Abbe refractometer and compared to Ref. [33]; liquid viscosities and densities are taken from the same source. These uncertainties combine to give the expected errors for $g, l^{*}$, and $\tau_{0}$ reported in Table I. In addition, two other aspects of the isotropic and anisotropic samples are not identical. First, the hydrodynamic self-interaction times quoted in Table I are different. This is unavoidable since the $93 \mathrm{~nm}$ spheres are smaller and since their solution has a higher viscosity; therefore, their $\tau_{\nu}$ is much smaller and their motion is more nearly diffusive over the time scales of our experiments. Second, the extrapolation length ratios that describe the diffuse photon boundary conditions are different. This is unavoidable since the two suspensions have different refractive indices,
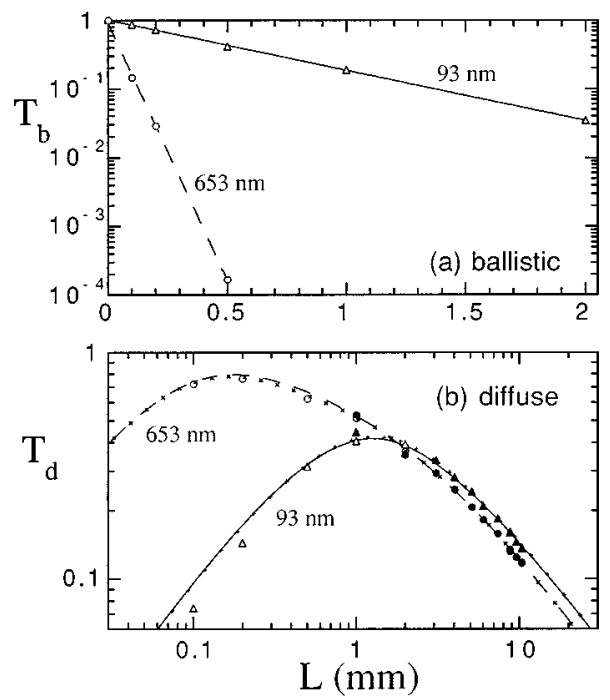

FIG. 10. Ballistic (top) and diffuse (bottom) transmission probability as a function of cell thickness. Triangles and circles respectively denote data for $93 \mathrm{~nm}$ spheres, which scatter light almost isotropically with $g=0.11$, and for $653 \mathrm{~nm}$ spheres, which scatter light very anisotropically with $g=0.90$. Open symbols are for relative measurements with an integrating sphere, and solid symbols for absolute; for all, the sample cells are held in air. The lines through the data in the upper plot represent fits to an exponential for the scattering length, $l_{S}$, while the curves through the data in the lower plot represent the prediction, Eq. (4.11). The small symbols are for random walk simulations with appropriate form factor and angledependent boundary reflectivity.

and hence different angle-dependent photon reflectivities at the suspension-wall interface. Values for $z_{e}$ are quoted in Table I for cases that the glass sample cells are held in air and in a water bath, where reflections at the wall-exterior interface are reduced. In spite of uncertainties in $l^{*}$ and $\tau_{0}$, and of differences in $\tau_{\nu}$ and $z_{e}$, the largest and most important difference between the $93 \mathrm{~nm}$ and $653 \mathrm{~nm}$ suspensions is their degree of scattering anisotropy. In analyzing data, our approach will be to treat only $l^{*}$ as an adjustable parameter, assuming that all other parameters in Table I are exact.

\section{B. Ballistic transmission}

Before examining the influence of scattering anisotropy on diffusing light spectroscopies, we further characterize the optical properties of the suspensions by measurements of the ballistic transmission probability. For this, we gently focus the collimated laser beam through samples contained in the precision spectrophotometer cells. A pinhole is placed at the focus so that scattered light is blocked and only the unscattered, ballistic component may reach the detector. The ballistic transmission probability is then obtained by normalizing to the signal from a cell containing pure water. The results displayed on semilogarithmic axes in Fig. 10(a) exhibit a clear exponential dependence on thickness, $T_{b}$ $=\exp \left(-L / l_{s}\right)$; this form neglects the contribution of ballistic photons, which reflect an even number of times before being transmitted since they are down by more than the square of boundary reflectivity at normal incidence, which is insignificant here. The fits shown in Fig. 10(a) give scattering lengths for the isotropic and anisotropic samples that differ by a 
TABLE II. Experimental values for $l^{*}$, given in units of $\mathrm{mm}$; the quoted error bars arise from uncertainties in fitting and normalization procedures.

\begin{tabular}{llll}
\hline \hline \multicolumn{1}{c}{ Method } & \multicolumn{1}{c}{ Bath } & Isotropic & Anisotropic \\
\hline Designed & & 0.52 & 0.52 \\
Ballistic transmission & Air & $0.66 \pm 0.02$ & $0.58 \pm 0.02$ \\
Diffuse transmission & Air & $0.59 \pm 0.02$ & $0.52 \pm 0.01$ \\
telegrapher DWS & Water & $0.67 \pm 0.01$ & $0.60 \pm 0.01$ \\
& Air & $0.68 \pm 0.01$ & $0.62 \pm 0.01$ \\
Standard DWS & Water & $0.68 \pm 0.01$ & $0.60 \pm 0.01$ \\
& Air & $0.69 \pm 0.01$ & $0.62 \pm 0.01$ \\
\hline \hline
\end{tabular}

factor of nearly ten, as expected by design. Using the calculated value of $g$, the transport mean free path may then be estimated as $l^{*}=l_{s} /(1-g)$; the results quoted in Table II are approximately equal and are close to the design values.

\section{Diffuse transmission}

We now consider the total diffusion transmission probability that a photon scattered out of the incident beam will exit, after any number of scattering events, from the opposite face of the sample. This can be measured absolutely, without reference to a control sample. Following our previous work [21], the illumination and collection optics are arranged so that the detection probability is independent of where on the sample face the transmitted or backscattered photons happen to emerge, as assumed in our predictions for the slab geometry. The measured light intensity is then recorded at finely spaced angles around the entire sample; the total transmitted and backscattered intensities, $I_{T}$ and $I_{B}$, may thus be found by integrating the resulting angular distributions. The final result for the diffuse transmission probability, $T_{d}=I_{T} /\left(I_{T}\right.$ $\left.+I_{B}\right)$, is displayed as solid symbols for both isotropic and anisotropic samples in Fig. 10(b). For thick samples, it decreases with increasing thickness in accord with the prediction $T_{d} \approx\left(1+z_{e}\right) /\left(L / l^{*}+2 z_{e}\right)$. Inverting this expression for $l^{*}$ using data from the five thickest samples, $L \geqslant 6 \mathrm{~mm}$, and averaging, gives an alternative characterization of the transport mean free path. The resulting values of $l^{*}$ shown in Table II are slightly smaller than those obtained from ballistic transmission, but are consistent with expectations.

To examine the thin samples contained in spectrophotometer cells, where anisotropy effects dominate the behavior of the diffuse transmission probability, we must employ a relative measuring scheme because the cell design prevents simultaneous observation of both transmitted and backscattered distributions. This is accomplished using a large diameter integrating sphere (Labsphere, North Sutton, NH). Samples are mounted at the input port, and the illuminating beam is gently focused so that unscattered light exits the sphere through a pinhole on the opposite side. The measured intensity at a photocell mounted on a third port is thus proportional to the diffuse transmission probability. The normalization constant is found by comparing results for $L=1$ and $2 \mathrm{~mm}$ cells with the absolute diffuse transmission probabilities obtained for rectangular cells of the same thickness.

The experimental results for $T_{d}$ are shown as open symbols in Fig. 10(b). For thick slabs, the isotropic and aniso- tropic samples exhibit nearly identical behavior, $T_{d} \approx(1$ $\left.+z_{e}\right) /\left(L / l^{*}+2 z_{e}\right)$, since their values of $l^{*}$ and of $z_{e}$ are so similar. For thin slabs, $L<2 \mathrm{~mm}$, by contrast, they have very different diffuse transmission probabilities, reaching a maximum near $L=2 l_{s}$ and decreasing for thinner slabs. This is similar to what was seen previously in the simulation test of Fig. 6, and is due to the great difference in their degrees of scattering anisotropy. In particular, thin anisotropic samples have a significantly larger $T_{d}$ both because their scattering length is ten times smaller, so that more photons scatter out of the incident beam, and because scattered photons are preferentially directed into the forward direction. For thin isotropic samples, by contrast, fewer photons scatter away from the incident beam, and those that do are as likely to be backscattered as transmitted. This behavior is captured very well by the prediction of Eq. (4.11), based on the treatment of scattering anisotropy by a discontinuity in diffuse photon concentration at the penetration point. These predictions are shown as curves in Fig. 10(b), using thick-sample inversion results for $l^{*}$ and numerically calculated values for $g, g^{\prime}$, $R_{b}$, and $z_{e}$ using the sample design parameters in Table I. None of these parameters is adjusted to fit the data. Simulation results using the appropriate Henyey-Greenstein form factor and angle-dependent boundary reflectivity are also included. For anisotropic samples, the prediction, simulation, and experiment are nearly indistinguishable as the slab thickness is varied widely from the single-scattering regime into the diffusive regime. For isotropic samples, similar agreement is found except that the experimental data are too low for the two thinnest slabs. This discrepancy is highly reproducible; given the close agreement between theory and simulation, we speculate that there remains an unknown source of systematic experimental error.

\section{Diffusing-wave spectroscopy}

We now turn to the main experiments for which our samples were designed: diffusing-wave spectroscopy study of intensity fluctuations caused by motion of the scattering sites. As for the diffuse transmission experiments, we will first analyze data from thick samples using traditional diffusion theory and then proceed to thin samples to observe the influence of scattering anisotropy. These measurements are performed using standard methods for samples contained in the extruded rectangular glass cells. Samples are illuminated with coherent light from the $\mathrm{Ar}^{+}$ion laser at near-normal incidence with no focusing. The diffusely transmitted or backscattered light is detected without imaging by a photomultiplier tube placed behind a $50 \mu \mathrm{m}$ diameter pinhole approximately $1 \mathrm{~m}$ from the sample. These scales were chosen so that the speckle and pinhole sizes are comparable; relative motion of the scattering sites then produces large fluctuations in the detected intensity. Our experimental geometry is equivalent to the traditional case of illuminating with a plane wave and imaging light, which emerges from a point [5], but it is much simpler and wastes far fewer photons [34]. Since neither geometry discriminates against photons according to lateral motion within the sample, they are both equivalent to the one-dimensional "plane-wave-in-everything-out" geometry assumed in our analysis. The PMT signal is amplified and discriminated so that each detected photon produces a 
square, TTL, pulse which can be fed directly to digital correlator board (ALV-5000, Langen/Hessen Germany) for realtime computation of the intensity autocorrelation function $\langle I(0) I(\tau)\rangle$. The result is related to the normalized electric field autocorrelation function, $g_{1}(\tau)$, by the Siegert relation $[35,36]$

$$
\frac{\langle I(0) I(\tau)\rangle}{\langle I\rangle^{2}}=B+\beta\left|g_{1}(\tau)\right|^{2},
$$

where $B$ is the baseline, which equals one if there are no drifts in the incident laser intensity, and $\beta$ is the intercept, which is less than one but increases with decreasing pinhole size and laser coherence.

It is common practice to test theories of DWS by nonlinear least-square fits to data in which both $l^{*}$ as well as the baseline and intercept are simultaneously adjusted [5,8,34]. This leads to outstanding fits but with results that vary with fitting interval [34], suggesting that errors in the predicted rate or shape of the correlation function are being masked by compensating errors in choice of fitting parameters. Here, we adopt a conservative approach in which the intercept and baseline are chosen once and for all without bias by predictions. In particular, the intercept $\beta$ is estimated by fitting the early-time data to a second degree polynomial. The baseline $B$ is estimated by averaging slightly less than one decade of data after the correlation disappears into the noise. The normalized field correlation function, and the corresponding statistical errors arising from uncertainties in baseline and intercept, are then found using the Siegert relation Eq. (6.3). To improve the signal-to-noise ratio, and to keep the baseline as close to one as possible, a line filter and polarizer oriented $90^{\circ}$ out of phase with respect to the incident beam are placed in front of the pinhole.

As a final experimental note, our DWS measurements were performed for samples either submerged in a temperature-controlled water bath, $T=21 \pm 0.2{ }^{\circ} \mathrm{C}$, or held in air at room temperature, $T=21 \pm 1^{\circ} \mathrm{C}$. Besides stabilizing the particle dynamics, the water bath serves to reduce boundary reflections so that $z_{e}$ for the isotropic and anisotropic samples becomes smaller and more nearly equal. This will be important since, according to Eq. (4.16), both the rate of decay and the curvature of the correlation function depend on the value of $z_{e}$.

After subtracting the baseline and dividing by the intercept, essentially raw correlation data are displayed in Fig. 11(a) for both transmission and backscattering geometries. As expected, by design, the results for isotropic and anisotropic samples exhibit nearly the same shapes and nearly the same time scales for decay. Also as expected, due to the DWS equation (2.7) and differences in path length distributions, only one decade in delay time is required for the primary decay of transmission data, while more than two decades are required for backscattering. Over these time scales, the particle motion is nearly diffusive, more so for the small spheres. This is seen in Fig. 11(b) by comparing the full Hinch prediction described above for $x=k^{2}\left\langle\Delta r^{2}(\tau)\right\rangle$ with its diffusive limit, $6 \tau / \tau_{0}$, using the calculated values for $\tau_{0}$ and $\tau_{\nu}$ in Table I.

All further analysis of the correlation data will be done in terms of behavior as a function of $x$, rather than of $\tau$, using

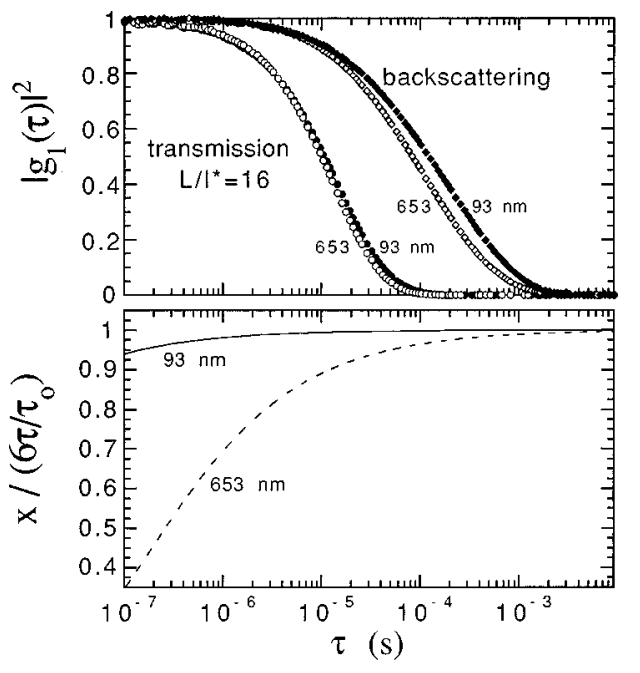

FIG. 11. DWS correlation function data vs delay time (upper plot) for isotropic and anisotropic samples. Note that the two transmission and the two backscattering correlation functions respectively decay with roughly the same shapes and time scales, as designed. The lower plot illustrates how the particle dynamics, $x$ $=k^{2}\left\langle\Delta r^{2}(\tau)\right\rangle$, exhibit a size-dependent long-time tail in the crossover to purely diffusive motion, $6 \tau / \tau_{0}$, at long times.

the Hinch prescription shown in Fig. 11(b). In other words, we assume that the designed polyball diameters and liquid viscosities, and hence the calculated values for $\tau_{0}$ and $\tau_{\nu}$, are all correct. This assumption can be tested two ways, first by fitting the correlation function in backscattering from the $L=10 \mathrm{~mm}$ samples, in air, to the approximate empirical form $g_{1}(x) \propto \exp \left(-\gamma \sqrt{x+x_{0}}\right)$; the $x_{0}$ term accounts for rounding at small $x$ due to loss of very long paths from either the finite size of the sample, absorption, or imperfect laser coherence. Since we have $x \cong 6 \tau / \tau_{0}$, any error in our assumption for $\tau_{0}$ would appear as an error in the fitted value of $\gamma$. Reasonable fits are found with $\gamma=1.40 \pm 0.02$ for the isotropic samples, $\gamma=1.80 \pm 0.05$ for the anisotropic samples, and $x_{0}=0.015$ for both. These values for $\gamma$ are smaller than the expectation $\gamma=1+z_{e}$ of Eq. (5.3), but are in good agreement with published experimental results on aqueous suspensions [12]. They are also in accord with the simulations results in Fig. 7, where anisotropic scattering leads to a faster decay.

We can further test the basic assumptions in our analysis by examining the early-time behavior in the transmission correlation functions, $-\ln \left[g_{1}(x)\right]=\Gamma_{1} x+O\left(x^{2}\right)$. Experimental values of the first cumulant, $\Gamma_{1}$, with respect to $x$, are thus deduced from asymptotic fits of $\ln \left[g_{1}(x)\right]$ to a second degree polynomial with an intercept of zero. These are displayed in Fig. 12(a) as $\sqrt{\Gamma_{1}^{-1} L^{2} / 6}$, because according to Eq. (5.2) this combination converges to $l^{*}$ in the limit of very thick slabs. Making this extrapolation with the predicted form of $\Gamma_{1}$ versus $L$ gives the $l^{*}$ values quoted in Table II. The results are independent of whether the diffusion or telegraphers predictions are used, since these theories agree for very thick samples. More significantly, the results are the same for data obtained for samples held in air and water baths, are consistent with the designed values, and happen to be in very close accord with the ballistic transmission measurements. Since we have $x \cong 6 \tau / \tau_{0}$ and $\Gamma_{1} \cong \widetilde{L^{2}} / 6$, giving $-\ln \left[g_{1}(x)\right]$ 


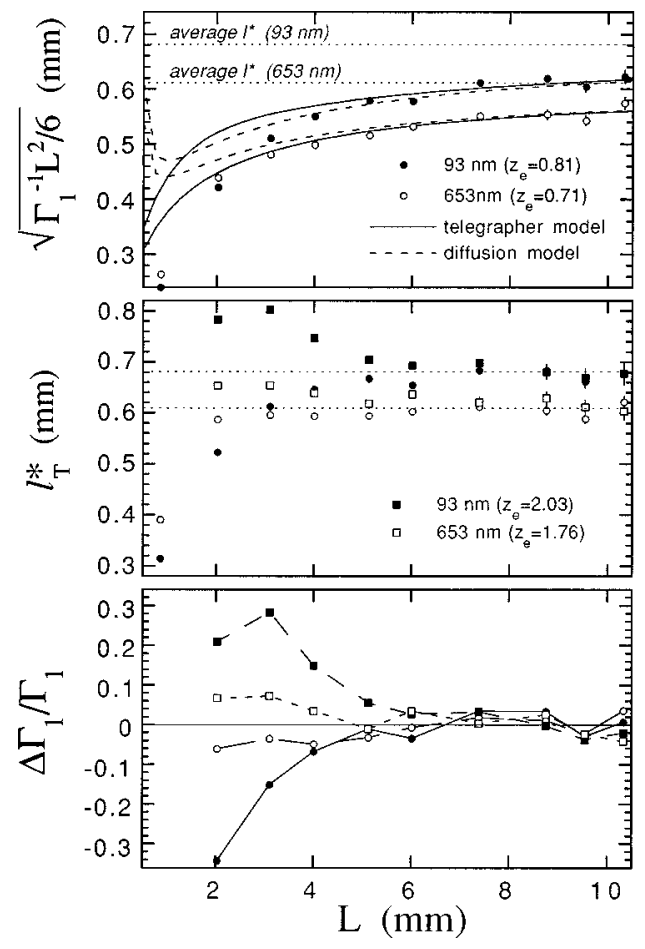

FIG. 12. First cumulant $\Gamma_{1}$ and corresponding inversion results for $l^{*}$ as a function of cell thickness; symbols denote data for different sphere size and bath conditions, as labeled by extrapolation length ratio. The value of $\Gamma_{1}$ is deduced from fits to $-\ln \left[g_{1}(x)\right]$ $=\Gamma_{1} x+O\left(x^{2}\right)$ for small $x$, while the value of $l^{*}$ displayed in the middle plot is deduced from $\Gamma_{1}$ using the telegrapher prediction of Eq. (4.14); error bars represent uncertainties from the cumulant fits. The dotted lines represent the average values of $l^{*}$ for $L>4 \mathrm{~mm}$, while the solid and dashed curves give the corresponding predictions for $\sqrt{\Gamma_{1}^{-1} L^{2} / 6}$ based on the telegrapher and diffusion models; note that $\sqrt{\Gamma_{1}^{-1} L^{2} / 6}$ converges to $l^{*}$ for very thick samples $(L$ $\left.\gg l^{*}\right)$. The bottom plot shows the fractional deviation of the predicted and measured cumulants.

$\cong\left(\tau L^{2}\right) /\left(\tau_{0} l^{* 2}\right)$, any error in our assumption for $\tau_{0}$ would appear as an error in the extrapolated values of $l^{*}$. Together with the backscattering and static transmission tests, this gives confidence in our analysis procedures and sample characterization.

Now that the samples are fully characterized, we may begin testing the telegrapher theory of DWS for thin samples. First consider again the cumulant data in Fig. 12(a). Both the telegrapher and diffusion predictions provide a good description for $L \geqslant 5 \mathrm{~mm}$, or $L / l^{*} \geqslant 8$. For thinner samples, the data and the two predictions progressively diverge. Note that our telegrapher theory more successfully captures the monotonic trend, and that the agreement is especially good for the anisotropic sample. This same comparison can be seen equivalently in the other two plots of Fig. 12, where we also include data for samples held in air. In the middle plot, we show an effective $l^{*}$ obtained by inverting the cumulant data using the telegrapher prediction. This display is most similar in spirit to previous experimental tests of DWS, e.g., Ref. [8] where an effective $l^{*}$ was obtained by fitting Eq. (2.8) to the entire decay. Here, we see that inversion results converge to a constant value of $l^{*}$ for thick samples. For thin samples, the results develop a systematic $L$

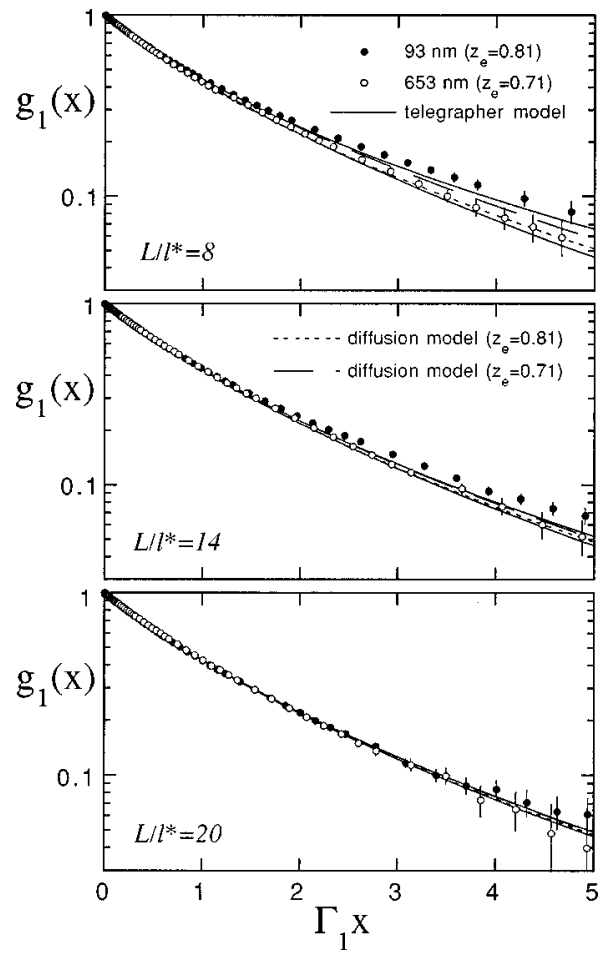

FIG. 13. DWS correlation function in transmission vs $\Gamma_{1} x$ for three slab thicknesses; error bars arise from uncertainty in the baseline. Plotted in this manner, the data (symbols) and predictions (curves) all exhibit the same small- $x$ behavior, $\ln \left[g_{1}(x)\right]=-\Gamma_{1} x$ $+O\left(x^{2}\right)$. For larger $x$, the upward curvature is greater for isotropic than for anisotropic samples. This distinction vanishes with increasing slab thickness in accord with the telegrapher model. Note that the curvature is slightly affected by the extrapolation length ratio, but opposite to the observed trends.

dependence as the theory becomes less accurate. This $L$ dependence is noticeably greater for the isotropic samples, whether or not submerged in a water bath. Lastly, the bottom plot in Fig. 12 shows the fractional deviation of the predicted cumulant from the measured one. Since the telegrapher predictions are evaluated using the average values of $l^{*}$ extracted from the upper and middle plots, the deviations in Fig. 12(c) all vanish, to within a systematic error of about \pm 0.03 , for very thick samples. For thin slabs, the isotropic samples develop a significant deviation as the theory becomes less accurate. Note that this display is most similar in spirit to the simulation test of Fig. 9. Furthermore, in fact, it is nearly identical in quantitative detail, except for the isotropic sample measured in air. This suggests that polarization and interference effects are negligible, and that the continuum approximation is therefore the dominant source of error in the telegrapher theory of DWS.

The final test of the telegrapher prediction concerns the shape, rather than the initial decay rate, of the correlation function. In Fig. 13, we display $g_{1}(x)$ data versus $\Gamma_{1} x$ for isotropic and anisotropic samples submerged in a water bath, along with predictions from telegrapher and diffusion theory. This is done on semilogarithmic axes for three different thicknesses. For small $x$, the data and predictions all match by construction, $\ln \left[g_{1}(x)\right] \cong-\Gamma_{1} x$. For larger $x$, the correlation functions all deviate from a simple exponential with some degree of upward curvature that is the actual quantity 
being tested in these plots. First observe that with increasing thickness, the data and predictions all converge to a shape with the same degree of curvature, as expected. We emphasize that the near-perfect agreement in Fig. 13 between theoretical and experimental shapes for thick samples, and also between telegrapher theory and experiment for thin samples, is not by fit: we have only matched the initial decay rates.

For thinner samples, the correlation function shapes in Fig. 13 depend increasingly both on the value of the boundary reflectivity, or equivalently on the extrapolation length ratio, $z_{e}$, and on the degree of scattering anisotropy, $g$. Note that the boundary reflectivities of the isotropic and anisotropic samples are close, but not equal; however, this cannot account for the observation that the isotropic samples have greater upward curvature. This is because the isotropic sample has the larger value of $z_{e}$, but according to both diffusion and telegrapher predictions with everything else being equal, this should lead to less curvature. The difference in degrees of scattering anisotropy is the real reason that the correlation functions for isotropic and anisotropic samples exhibit different curvatures; the difference in boundary reflectivity merely reduces this effect. We therefore believe that the shape differences apparent in Fig. 13 are the first experimental demonstration of the influence of scattering anisotropy. The observed trend is, of course, not accounted for, or correctly predicted by, standard diffusion theory. It is, however, captured quantitatively to a high degree by our telegrapher theory. As seen in Fig. 13, the predicted curvature is systematically not quite as great as measured. A similar deviation was also seen in the simulation results of Fig. 8, and is thus attributed to the continuum approximation.

\section{CONCLUSIONS}

It is now straightforward to estimate the influence of ballistic transport and of anisotropic scattering within an analytically tractable transport theory. As argued earlier [17], the former is accomplished for scattered photons by a telegrapher equation with suitable prescriptions for boundary conditions and the emerging flux. As argued here, it is accomplished for unscattered photons within the incident beam by integrating over source planes and summing over reflections. Also as argued here, the influence of scattering anisotropy is estimated by introducing a discontinuity in photon concentration at the source plane in proportion to the degree of scattering anisotropy. We have demonstrated the validity of this approach by random walk simulations, and the accuracy of the resulting DTS and DWS predictions by both simulation and experiment.

The bottom line for DTS is that we can now predict the diffuse transmission probability through a slab to an accuracy of better than a few percent. This is true for any degree of scattering anisotropy, i.e., for any angle-dependent scattering form factor, and for any angle-dependent boundary reflectivity. Furthermore, it is true for any slab thickness, from single scattering, through intermediate, and into the diffusive regimes. To our knowledge, this is unprecedented in an exactly solvable theory. Here we have considered only the case of normal incidence with no absorption, but these variations are straightforward to include within our approach; other geometries can also be handled. This should be useful for analyzing transmission data in terms of the three fundamental optical length scales, $l^{*}, l_{s}$, and $l_{a}$, set by the structure of the medium.

The bottom line for DWS is that we can now predict the influence of scattering anisotropy on the shape of the correlation function. This extends the utility of DWS to thinner samples and to deeper decays of $g_{1}(x)$, and should thus be useful for more accurately analyzing data in terms of the time-dependent dynamics $\left\langle\Delta r^{2}(\tau)\right\rangle$ of the scattering sites. While our photon transport theory may be accurate, for very thin slabs the continuum approximation $Y \cong S$ of Eq. (2.6) fails altogether because of the presence of snakelike photon paths crossing the slab with $S \cong L$ but with $Y \cong 0$ [10]. Therefore, by contrast with DTS, our predictions for DWS cannot be applied for thicknesses below $L \cong 2 l^{*}$. The primary symptom is a greater upward curvature in the shape of $g_{1}(x)$ than predicted, because $P(Y)$ is broader than $P(S)$. To artificially introduce more small paths into the theoretical path length distribution, we propose simply to set $D_{0}^{2}=0$ in Eq. (4.14). This means purposely neglecting causality and the influence of ballistic propagation in order to compensate for error in the continuum approximation. The DWS predictions are then simpler and, as seen in Fig. 9, even more accurate. Until the continuum approximation can be improved upon, we therefore recommend Eq. (4.14) with $D_{0}^{2}=0$ as the best analytic means of analyzing DWS data.

\section{ACKNOWLEDGMENTS}

Helpful conversations with B. J. Ackerson, A. D. Gopal, D. J. Pine, J. Rudnick, D. A. Weitz, and A. G. Yodh are gratefully acknowledged. This work was supported in part by NASA Grant No. NAG3-1419 and by NSF Grant No. DMR9623567.
[1] Diffusing Photons in Turbid Media, edited by A. G. Yodh, B. Tromberg, E. Sevick-Muraca, and D. J. Pine, special issue of J. Opt. Soc. Am. A 14, 136 (1997)].

[2] D. A. Weitz and D. J. Pine, in Dynamic Light Scattering: The Method and some Applications, edited by W. Brown (Claredon Press, Oxford, 1993), p. 652.

[3] P. D. Kaplan, A. D. Dinsmore, A. G. Yodh, and D. J. Pine, Phys. Rev. E 50, 4827 (1994).

[4] G. Maret and P. E. Wolf, Z. Phys. B 65, 409 (1987).
[5] D. J. Pine, D. A. Weitz, P. M. Chaikin, and E. Herbolzheimer, Phys. Rev. Lett. 60, 1134 (1988).

[6] M. J. Stephen, Phys. Rev. B 37, 1 (1988).

[7] K. M. Yoo, F. Liu, and R. R. Alfano, Phys. Rev. Lett. 64, 2647 (1990).

[8] P. D. Kaplan, M. H. Kao, A. G. Yodh, and D. J. Pine, Appl. Opt. 32, 3828 (1993).

[9] D. J. Durian, Phys. Rev. E 50, 857 (1994).

[10] D. J. Durian, Phys. Rev. E 51, 3350 (1995). 
[11] F. C. MacKintosh and S. John, Phys. Rev. B 40, 2383 (1989).

[12] F. C. MacKintosh, J. X. Zhu, D. J. Pine, and D. A. Weitz, Phys. Rev. B 40, 9342 (1989).

[13] B. J. Ackerson, R. L. Dougherty, N. M. Reguigui, and U. Nobbmann, J. Thermophys. Heat Transfer 6, 577 (1992).

[14] R. L. Dougherty, B. J. Ackerson, N. M. Reguigui, F. DorrNowkoorani, and U. Nobbmann, J. Quant. Spectrosc. Radiat. Transf. 52, 713 (1994).

[15] A. A. Middleton and D. S. Fisher, Phys. Rev. B 43, 5934 (1991).

[16] D. J. Durian, Physica A 229, 218 (1996).

[17] D. J. Durian and J. Rudnick, J. Opt. Soc. Am. A 14, 235 (1997).

[18] K. M. Case and P. F. Zweifel, Linear Transport Theory (Addison-Wesley, New York, 1967).

[19] J. J. Duderstadt and W. R. Martin, Transport Theory (Wiley, New York, 1979).

[20] J. X. Zhu, D. J. Pine, and D. A. Weitz, Phys. Rev. A 44, 3948 (1991)

[21] M. U. Vera and D. J. Durian, Phys. Rev. E 53, 3215 (1996).

[22] S. Chandrasekhar, Radiative Transfer (Dover Publications, New York, 1960).

[23] C. F. Bohren, Am. J. Phys. 55, 524 (1987).
[24] L. G. Henyey and J. L. Greenstein, Astrophys. J. 93, 70 (1941).

[25] H. C. van de Hulst, Light Scattering by Small Particles (Dover, New York, 1957).

[26] C. F. Bohren and D. R. Huffman, Absorption and Scattering of Light by Small Particles (Wiley, New York, 1983).

[27] G. K. Batchelor, J. Fluid Mech. 74, 1 (1976).

[28] W. van Megen, S. M. Underwood, R. H. Ottewill, N. S. J. Williams, and P. N. Pusey, Faraday Discuss. Chem. Soc. 83, 47 (1987)

[29] A. van Veluwen, H. N. W. Lekkerkerker, C. G. de Kruif, and A. Vrij, Faraday Discuss. Chem. Soc. 83, 59 (1987).

[30] J. X. Zhu, D. J. Durian, J. Muller, D. A. Weitz, and D. J. Pine, Phys. Rev. Lett. 68, 2559 (1992).

[31] E. J. Hinch, J. Fluid Mech. 72, 499 (1975).

[32] American Institute of Physics Handbook, edited by D. E. Grey (McGraw-Hill, New York, 1972).

[33] Glycerol, edited by C. S. Miner and N. N. Dalton, Monograph Series of the American Chemical Society Vol. 117 (Reinhold, New York, 1953).

[34] D. J. Durian, Appl. Opt. 34, 7100 (1995).

[35] B. J. Berne and R. Pecora, Dynamic Light Scattering, with Applications to Chemistry, Biology, and Physics (Wiley, New York, 1976).

[36] T. Bellini, M. A. Glaser, N. A. Clark, and V. Degiorgio, Phys. Rev. A 44, 5215 (1991). 